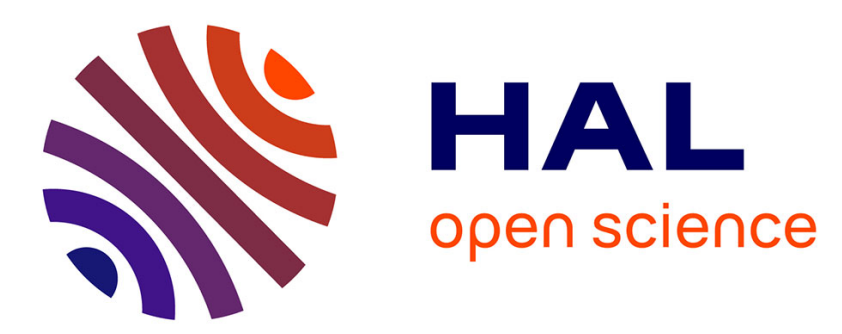

\title{
Exact Boundary Controllability of a System of Mixed Order with Essential Spectrum
}

\author{
F. Ammar Khodja, Karine Mauffrey, Arnaud Munch
}

\section{To cite this version:}

F. Ammar Khodja, Karine Mauffrey, Arnaud Munch. Exact Boundary Controllability of a System of Mixed Order with Essential Spectrum. SIAM Journal on Control and Optimization, 2011, 49 (4), pp.1857-1879. 10.1137/090777712 . hal-00864107

\section{HAL Id: hal-00864107 https://hal.science/hal-00864107}

Submitted on 20 Sep 2013

HAL is a multi-disciplinary open access archive for the deposit and dissemination of scientific research documents, whether they are published or not. The documents may come from teaching and research institutions in France or abroad, or from public or private research centers.
L'archive ouverte pluridisciplinaire HAL, est destinée au dépôt et à la diffusion de documents scientifiques de niveau recherche, publiés ou non, émanant des établissements d'enseignement et de recherche français ou étrangers, des laboratoires publics ou privés. 


\title{
EXACT BOUNDARY CONTROLLABILITY OF A SYSTEM OF MIXED ORDER WITH ESSENTIAL SPECTRUM*
}

\author{
F. AMMAR KHODJA ${ }^{\dagger}$, K. MAUFFREY ${ }^{\dagger}$, AND A. MÜNCH
}

\begin{abstract}
We address in this work the exact boundary controllability of a linear hyperbolic system of the form $u^{\prime \prime}+A u=0$ with $u=\left(u_{1}, u_{2}\right)^{T}$ posed in $(0, T) \times(0,1)^{2}$. A denotes a self-adjoint operator of mixed order that usually appears in the modelization of a linear elastic membrane shell. The operator $A$ possesses an essential spectrum which prevents the exact controllability from holding uniformly with respect to the initial data $\left(u^{0}, u^{1}\right)$. We show that the exact controllability holds by one Dirichlet control acting on the first variable $u_{1}$ for any initial data $\left(u^{0}, u^{1}\right)$ generated by the eigenfunctions corresponding to the discrete part of the spectrum of $A$. The proof relies on a suitable observability inequality obtained by way of a full spectral analysis and the adaptation of an Inghamtype inequality for the Laplacian in two spatial dimensions. This work provides a nontrivial example of a system controlled by a number of controls strictly lower than the number of components. Some numerical experiments illustrate our study.
\end{abstract}

Key words. boundary controllability, essential spectrum, Ingham inequality, mixed order operator

AMS subject classifications. 35L20, 93B05, 93B07, 93B60, 93C20

DOI. $10.1137 / 090777712$

1. Introduction and problem statement. Let $\Omega=(0,1)^{2}$ and $\Gamma$ be the part of $\partial \Omega$ defined by $\Gamma=\{(x, y) \in \partial \Omega, x y=0\}$. Let $T$ be a positive real number and $a, \alpha$ be two real numbers such that $a>\alpha^{2}>0$ and $\sqrt{a-\alpha^{2}} / \pi \notin \mathbb{N}^{*}$. We analyze in this work the exact boundary controllability of the following system in $u=\left(u_{1}, u_{2}\right)^{T}$ :

$$
\begin{cases}u^{\prime \prime}+A u=0 & \text { in } Q_{T}=\Omega \times(0, T), \\ u_{1}=v 1_{\Gamma} & \text { on } \Sigma_{T}=\partial \Omega \times(0, T), \\ \left(u(\cdot, 0), u^{\prime}(\cdot, 0)\right)=\left(u^{0}, u^{1}\right) & \text { in } \Omega,\end{cases}
$$

where

$$
A=\left(\begin{array}{cc}
-\Delta & -\alpha \partial_{x} \\
\alpha \partial_{x} & a
\end{array}\right)
$$

The study of this system is motivated by the following in $u=\left(u_{1}, u_{2}, u_{3}\right)^{T}$ :

$$
\begin{cases}u^{\prime \prime}+\mathcal{A} u=0 & \text { in } Q_{T}, \\ \left(u_{1}, u_{2}\right)=\left(v_{1}, v_{2}\right) 1_{\Gamma} & \text { on } \Sigma_{T}, \\ \left(u(\cdot, 0), u^{\prime}(\cdot, 0)\right)=\left(u^{0}, u^{1}\right) & \text { in } \Omega\end{cases}
$$

${ }^{*}$ Received by the editors November 18, 2009; accepted for publication (in revised form) May 12, 2011; published electronically August 23, 2011.

http://www.siam.org/journals/sicon/49-4/77771.html

$\dagger$ Laboratoire de Mathématiques de Besançon, UMR CNRS 6623, Université de FrancheComte, 16 route de Gray, 25030 Besançon Cedex, France (farid.ammar-khodja@univ-fcomte.fr, karine.mauffrey@univ-fcomte.fr). The work of the second author was partially supported by grants ANR-07-JCJC-0139-01 (Agence nationale de la recherche, France) and by Région de Franche-Comté (France).

${ }^{\ddagger}$ Laboratoire de Mathématiques de Clermont-Ferrand, UMR CNRS 6620, Université Blaise Pascal, Campus des Cézeaux, 63177 Aubière Cedex, France (arnaud.munch@math.univ-bpclermont.fr). This author's work was partially supported by grants ANR-07-JCJC-0139-01 (Agence nationale de la recherche, France) and 08720/PI/08 from Fundacíon Séneca (Gobernio regional de Murcia, Spain). 
where the operator

$$
\mathcal{A}=\left(\begin{array}{ccc}
-a \partial_{x x}^{2}-c \partial_{y y}^{2} & -(b+c) \partial_{x y}^{2} & -a r^{-1} \partial_{x} \\
-(b+c) \partial_{x y}^{2} & -c \partial_{x x}^{2}-a \partial_{y y}^{2} & -b r^{-1} \partial_{y} \\
r^{-1} a \partial_{x} & r^{-1} b \partial_{y} & r^{-2} a
\end{array}\right)
$$

is used to model a cylindrical membrane elastic shell (see [20]). Here $a=8 \mu(\lambda+$ $\mu) /(\lambda+2 \mu), b=4 \lambda \mu /(\lambda+2 \mu)$ and $c=2 \mu$, where $\lambda, \mu>0$ denote the Lamé coefficients and $r^{-1}>0$ - the coupling parameter between the tangential displacement $\left(u_{1}, u_{2}\right)$ and the normal displacement $u_{3}$ of the shell - denotes the curvature of the cylinder. This mixed order and formally self-adjoint operator, when associated with homogeneous Dirichlet boundary conditions on the first two components, enters in the framework of [6]. It possesses an essential spectrum $\sigma_{\text {ess }}(\mathcal{A})$ that can be computed using [7]: precisely, $\sigma_{\text {ess }}(\mathcal{A})=\left[0,2 r^{-2}(3 \lambda+2 \mu) /(\lambda+\mu)\right]$. Therefore, the spectrum of $\mathcal{A}$ is composed of two distinct parts, the essential spectrum plus a discrete spectrum with asymptotic behavior equal, up to some constant, to the spectrum of $-\Delta$. The difficulty here is that the discrete spectrum is not known explicitly. In this paper, instead of $\mathcal{A}$ we consider the operator $A$ which presents the same structure yet is simpler and has a spectrum that can be explicitly computed.

We set $H=L^{2}(\Omega) \times L^{2}(\Omega)$ (equipped with the natural scalar product $\langle\cdot, \cdot\rangle_{H}$ ) and $H_{1 / 2}=H_{0}^{1}(\Omega) \times L^{2}(\Omega)$. Let $H_{-1 / 2}$ denote the dual of $H_{1 / 2}$ with respect to the pivot space $H$. System (1.1) is said to be exactly controllable at time $T>0$ if for any initial data $\left(u^{0}, u^{1}\right) \in H \times H_{-1 / 2}$ and any target $\left(u_{T}^{0}, u_{T}^{1}\right) \in H \times H_{-1 / 2}$ there exists a control function $v$ in a suitable space, such that the unique solution $u=\left(u_{1}, u_{2}\right)^{T}$ of system (1.1) satisfies

$$
\left(u(\cdot, T), u^{\prime}(\cdot, T)\right)=\left(u_{T}^{0}, u_{T}^{1}\right) \quad \text { in } \Omega .
$$

We point out that the variable $u_{2}$ in system (1.1) is free of any condition on the boundary $\partial \Omega$. In particular, system (1.1) provides a nontrivial example for which the number of controls is strictly lower than the number of components (for other examples, see, for instance, [22] and [11]).

As usual, the controllability issue is equivalent to an observability inequality that we will rigorously prove to be

$$
\left\|\left(\Phi^{0}, \Phi^{1}\right)\right\|_{H_{1 / 2} \times H}^{2} \leq C \int_{0}^{T} \int_{\Gamma}\left(\frac{\partial \varphi}{\partial \nu}+\alpha \psi \nu_{1}\right)^{2} d \sigma d t
$$

$\left(\nu=\left(\nu_{1}, \nu_{2}\right)\right.$ denotes the unit outward normal to $\left.\Gamma\right)$ for any initial data $\left(\Phi^{0}, \Phi^{1}\right)$ belonging to $H_{1 / 2} \times H$, for the following homogeneous adjoint system in $\Phi=(\varphi, \psi)^{T}$ :

$$
\begin{cases}\Phi^{\prime \prime}+A \Phi=0 & \text { in } Q_{T} \\ \varphi=0 & \text { on } \Sigma_{T}, \\ \left(\Phi(\cdot, 0), \Phi^{\prime}(\cdot, 0)\right)=\left(\Phi^{0}, \Phi^{1}\right) & \text { in } \Omega .\end{cases}
$$

The observation zone $\Gamma$ is defined so that the triplet $(\Omega, \Gamma, T)$ satisfies the geometric optic condition. 
The originality and difficulty of the - apparently simple - system (1.3) are related to the fact that $A$ is a mixed order operator and, therefore, possesses a nonempty essential spectrum $\sigma_{\text {ess }}(A)$, as shown in [6] in a general situation. As a consequence, the observability does not hold uniformly with respect to the data $\left(\Phi^{0}, \Phi^{1}\right)$. Precisely, in [10] the authors exhibit Weil sequences, associated with some elements of $\sigma_{\text {ess }}(A)$ for which the observability inequality (1.2) is not true. The observability is therefore expected only, roughly speaking, in the orthogonal of some space related to the essential spectrum. To our knowledge, up to now the only way to address this kind of problem is based on spectral analysis and an Ingham-type approach and can be used to prove the observability for the discrete part of the spectrum, provided some spectral gap conditions (see [12]). In that framework, the existing literature mainly concerns the controllability of dynamical systems modeling the vibrations of an elastic membrane shell, where precisely mixed order and self-adjoint operators appear (we refer to [20] for a detailed spectral analysis). We also mention $[8,9]$, where the controllability of a hemispherical cap is studied using a nonharmonic spectral analysis. The analysis, reduced to the one spatial dimension by axial symmetry, exhibits the loss of uniform observability due to the essential spectrum composed of a single positive element. A similar study is performed in [2] for a nonuniform elliptic operator $A$ for which $0 \in \sigma_{\text {ess }}(A)$. We also refer the reader to Chapter 5 of [13] for results based on some recent extensions of Ingham-type inequalities. For systems of this kind, the uniform partial controllability, which consists of driving to rest only a restricted number of components, is proved in [14]. The observability is obtained by a so-called spectral compensation argument, which states that the bad behavior of the part of the spectrum which accumulates to $\sigma_{\text {ess }}(A)$ is somehow compensated by the suitable gap of the discrete part. In a different context, we also mention $[3,17,19]$ for the controllability of systems with spectral accumulation point.

The paper is organized as follows. In section 2, after introducing some spaces in connection with spectral properties of the operator $A$, we set our main results. In section 3, we establish the well-posedness of (1.1) for $v$ and initial data $\left(u^{0}, u^{1}\right)$ in suitable spaces. We divide section 4 into three parts. The first is devoted to the analysis of the adjoint system and to the formulation of the observability inequality as (1.2). In the second part, we prove the observability for any initial data $\left(\Phi^{0}, \Phi^{1}\right)$ belonging to a closed subspace of $H_{1 / 2} \times H$ spanned by eigenfunctions of $A$ associated with the isolated eigenvalues of $A$ with finite multiplicity. The key point is that these eigenvalues enjoy gap properties similar to those of the eigenvalues of $-\Delta$ with the Dirichlet boundary condition used in [18]. On the contrary, the third part of section 4 exhibits the lack of observability in spaces related to the essential spectrum. In particular, by numerical approximation, we check that the corresponding observability constant is not uniformly bounded with respect to $\left(\Phi^{0}, \Phi^{1}\right)$. Section 5 concludes this work with some remarks and open problems.

2. Preliminaries and main results. We recall that the eigenvalues and normalized eigenfunctions of the operator $-\Delta$ with domain $H^{2}(\Omega) \cap H_{0}^{1}(\Omega)$ are given by $\mu_{p q}=\left(p^{2}+q^{2}\right) \pi^{2}$ and $\varphi_{p q}(x, y)=2 \sin (p \pi x) \sin (q \pi y)$ (for $(p, q)$ in $\mathbb{N}^{*} \times \mathbb{N}^{*}$ and $\left.(x, y) \in \Omega=(0,1)^{2}\right)$.

The natural operator that occurs when we transform (1.3) into a second order differential equation is $A=\left(\begin{array}{cc}-\Delta \partial_{x} & -\alpha \partial_{x}\end{array}\right)$ in $H=L^{2}(\Omega) \times L^{2}(\Omega)$ with domain $D(A)=\left(H^{2}(\Omega) \cap H_{0}^{1}(\Omega)\right) \times D\left(\partial_{x}\right)$. Here $\partial_{x}$ is considered an unbounded operator in $L^{2}(\Omega)$. We can prove that this operator $A$ is not closed in $H$. Therefore we have 
to consider its closure, which is still denoted by $A$ and defined by (see [1])

$$
\begin{gathered}
A\left(\begin{array}{c}
\varphi \\
\psi
\end{array}\right)=\left(\begin{array}{c}
-\Delta\left(\varphi+\alpha \Delta^{-1} \partial_{x} \psi\right) \\
\alpha \partial_{x} \varphi+a \psi
\end{array}\right) \\
D(A)=\left\{(\varphi, \psi)^{T} \in H_{0}^{1}(\Omega) \times L^{2}(\Omega) / \varphi+\alpha \Delta^{-1} \partial_{x} \psi \in H^{2}(\Omega)\right\} .
\end{gathered}
$$

We introduce the following notation.

Notation 2.1. For $p, q \geq 1$, we set

$$
\begin{aligned}
& \lambda_{p, q}^{ \pm}=\frac{1}{2}\left(\mu_{p q}+a \pm \sqrt{\left(\mu_{p q}-a\right)^{2}+4 \alpha^{2} p^{2} \pi^{2}}\right), \mu_{p q}=\left(p^{2}+q^{2}\right) \pi^{2}, \\
& \varphi_{p q}(x, y)=2 \sin (p \pi x) \sin (q \pi y), \psi_{p q}(x, y)=2 \cos (p \pi x) \sin (q \pi y), \\
& e_{p, q}^{ \pm}=\left(\frac{\left(\lambda_{p, q}^{ \pm}-a\right)}{\sqrt{\left(\lambda_{p, q}^{ \pm}-a\right)^{2}+\alpha^{2} p^{2} \pi^{2}}} \varphi_{p q}, \frac{\alpha p \pi}{\sqrt{\left(\lambda_{p, q}^{ \pm}-a\right)^{2}+\alpha^{2} p^{2} \pi^{2}}} \psi_{p q}\right)^{T}, \\
& e_{q}(x, y)=(0, \sqrt{2} \sin (q \pi y))^{T} .
\end{aligned}
$$

Straightforward computations show that for every $(p, q) \in \mathbb{N}^{*} \times \mathbb{N}^{*}$, we have $A e_{p, q}^{ \pm}=\lambda_{p, q}^{ \pm} e_{p, q}^{ \pm}, A e_{q}=a e_{q}$, and for each $\lambda \notin\{a\} \cup\left\{\lambda_{p, q}^{+}\right\}_{p, q \geq 1} \cup\left\{\lambda_{p, q}^{-}\right\}_{p, q \geq 1}$, $\operatorname{Ker}(A-\lambda I)=\{0\}$. In other words, the sets of eigenvalues and associated eigenfunctions of $A$ are $\{a\} \cup\left\{\lambda_{p, q}^{+}\right\}_{p, q \geq 1} \cup\left\{\lambda_{p, q}^{-}\right\}_{p, q \geq 1}$ and $\left\{e_{q}\right\}_{q \geq 1} \cup\left\{e_{p, q}^{+}\right\}_{p, q \geq 1} \cup\left\{e_{p, q}^{-}\right\}_{p, q \geq 1}$, respectively. It is also easy to check that $\lambda_{p, q}^{+} \sim_{\|(p, q)\| \rightarrow+\infty} \mu_{p q}$ (the order of approximation is $\left.\lambda_{p, q}^{+}=\mu_{p q}+\frac{\alpha^{2} p^{2} \pi^{2}}{\mu_{p q}-a}+\mathrm{O}\left(\frac{p^{4}}{\mu_{p q}^{3}}\right)\right)$, and $\lambda_{p, q}^{-} \leq a<\lambda_{1,1}^{+} \leq \lambda_{p, q}^{+}$for all $p, q \geq 1$. Thus all the $\lambda_{p, q}^{+}$are isolated eigenvalues (of finite multiplicity). Using the fact that the families $\left\{\varphi_{p q}\right\}_{p, q \geq 1}$ and $\left\{\psi_{p q}\right\}_{p \geq 0, q \geq 1}$ are two orthonormal bases of $L^{2}(\Omega)$, we can easily check that the family $\mathcal{B}=\left\{e_{q}\right\}_{q \geq 1} \cup\left\{e_{p, q}^{+}\right\}_{p, q \geq 1} \cup\left\{e_{p, q}^{-}\right\}_{p, q \geq 1}$ forms an orthonormal basis of $H=L^{2}(\Omega) \times L^{2}(\Omega)$.

We refer to [5] for the definition of the essential spectrum $\sigma_{\text {ess }}(A)$ of $A$. As a consequence of the results in $[6]$, we have $\sigma_{\text {ess }}(A)=\left[a-\alpha^{2}, a\right]$. Besides, we can check that the set of accumulation points of the sequence $\left(\lambda_{p, q}^{-}\right)_{p, q}$ is exactly $\sigma_{e s s}(A)$. Figure 2.1 summarizes these properties.

Remark 2.2. The asymptotic behavior of $\left(\lambda_{p, q}^{+}\right)_{p, q}$ is in agreement with [7], where it is shown that the asymptotic behavior of $\sigma(A)$ is related to the spectrum of the principal part of $A$, which in our case is $-\Delta$.

The operator $A$ defined in (2.1) is self-adjoint in $H$ and positive; this last assertion

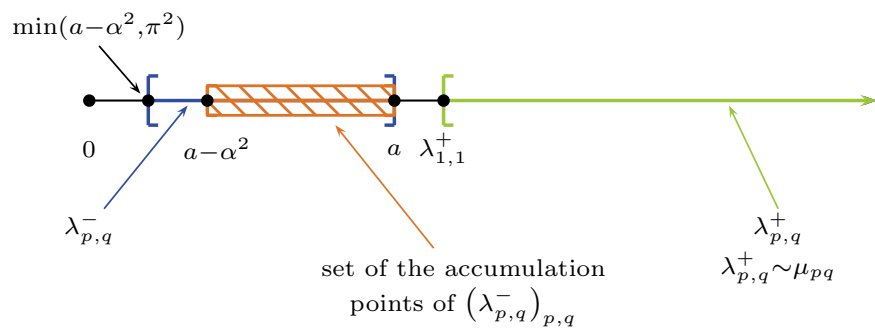

FIG. 2.1. Distribution of the spectrum of $A$ along $\mathbb{R}$.

Copyright $@$ by SIAM. Unauthorized reproduction of this article is prohibited. 
comes from the formula

$$
\langle A u, u\rangle_{H}=\int_{\Omega}\left[\left(\partial_{x} u_{1}+\alpha u_{2}\right)^{2}+\left(a-\alpha^{2}\right) u_{2}^{2}+\left(\partial_{y} u_{1}\right)^{2}\right] d x d y
$$

(for $u=\left(u_{1}, u_{2}\right)^{T} \in D(A)$ ) and the assumption $a>\alpha^{2}$.

For any $\delta \in \mathbb{R}_{+}$, we recall that the operator $A^{\delta}$ is defined by

$$
\begin{gathered}
A^{\delta}=\sum_{p, q \geq 1}\left(\lambda_{p, q}^{+}\right)^{\delta}\left\langle\cdot, e_{p, q}^{+}\right\rangle_{H} e_{p, q}^{+}+\sum_{p, q \geq 1}\left(\lambda_{p, q}^{-}\right)^{\delta}\left\langle\cdot, e_{p, q}^{-}\right\rangle_{H} e_{p, q}^{-}+a^{\delta} \sum_{q \geq 1}\left\langle\cdot, e_{q}\right\rangle_{H} e_{q}, \\
D\left(A^{\delta}\right)=\left\{\phi \in H, \sum_{p, q \geq 1}\left(\lambda_{p, q}^{+}\right)^{2 \delta}\left\langle\phi, e_{p, q}^{+}\right\rangle_{H}^{2}+\sum_{p, q \geq 1}\left(\lambda_{p, q}^{-}\right)^{2 \delta}\left\langle\phi, e_{p, q}^{-}\right\rangle_{H}^{2}+a^{2 \delta} \sum_{q \geq 1}\left\langle\phi, e_{q}\right\rangle_{H}^{2}<\infty\right\} .
\end{gathered}
$$

Since $\left(\lambda_{p, q}^{-}\right)_{p, q}$ is bounded, $D\left(A^{\delta}\right)=\left\{\phi \in H, \sum_{p, q \geq 1}\left(\lambda_{p, q}^{+}\right)^{2 \delta}\left\langle\phi, e_{p, q}^{+}\right\rangle_{H}^{2}<\infty\right\}$. In what follows, we will set

$$
H_{\delta}=D\left(A^{\delta}\right), \quad \delta \geq 0 .
$$

The operator $A$ is a bounded operator from $D(A)$, equipped with the graph norm, to $H$. It is well known that $A$ can be extended to a bounded operator from $H$ to $D(A)^{\prime}$, the dual space of $D(A)$ with respect to the pivot space $H$. We continue to denote this extension by $A$, and, thus, $A$ can be seen as an unbounded self-adjoint operator on $D(A)^{\prime}$ with domain $H$. $A$ is also a unitary operator from $D(A)$ to $H$ and from $H$ to $D(A)^{\prime}$. In what follows we will set

$$
H_{-1}=D(A)^{\prime} .
$$

The other extension of $A$ that we will use later is the following. $A$ can also be extended to a unitary operator from $D\left(A^{1 / 2}\right)$, equipped with the graph norm, to $D\left(A^{1 / 2}\right)^{\prime}$, the dual space of $D\left(A^{1 / 2}\right)$ with respect to the pivot space $H$. We will set

$$
H_{1 / 2}=D\left(A^{1 / 2}\right), \quad H_{-1 / 2}=D\left(A^{1 / 2}\right)^{\prime} .
$$

For details on these extensions, see, for instance, [21]. The last notation we will need is the following:

$$
H^{ \pm}=\operatorname{span}\left(\left\{e_{p, q}^{ \pm}, p, q \geq 1\right\}\right), \quad H^{a}=\operatorname{span}\left(\left\{e_{q}, q \geq 1\right\}\right),
$$

and for $\delta \in \mathbb{R}$,

$$
H_{\delta}^{ \pm}=H_{\delta} \cap H^{ \pm}, \quad H_{\delta}^{a}=H_{\delta} \cap H^{a} .
$$

We are now ready to set our main result.

Theorem 2.3. Let $\gamma=\frac{\pi \sqrt{\pi}}{4 \sqrt{2 \pi+|\alpha|}}$ and $T_{0}=\frac{2 \pi}{\gamma} \sqrt{1+2 \frac{\left(\lambda_{1,1}^{+}-a+\alpha^{2}\right)^{2}}{\left(\lambda_{1,1}^{+}-a\right)^{2}}}$. Assume that $a \leq 2 \pi^{2}$. For any $T>T_{0}$, any initial data $\left(u^{0}, u^{1}\right) \in H^{+} \times H_{-1 / 2}^{+}$, and any target $\left(u_{T}^{0}, u_{T}^{1}\right) \in H^{+} \times H_{-1 / 2}^{+}$, there exist control functions $v \in L^{2}(\Gamma \times(0, T))$ such that the unique solution $u$ of system (1.1) satisfies $u(\cdot, T)=u_{T}^{0}$ and $u^{\prime}(\cdot, T)=u_{T}^{1}$ in $\Omega$.

The significant novelty of this result with respect to the literature mentioned in the introduction is that it concerns two spatial dimensions. The proof of this theorem is much less straightforward (than for the one-dimensional situation). It relies on the 
adaptation to our problem of a recent Ingham-type theorem due to Mehrenberger for the wave equation (see [18]). The restriction to the closed subspace $H^{+} \times H_{-1 / 2}^{+}$is justified by the following proposition.

Proposition 2.4. For any $T>0$ and any $\epsilon>0$, there exist initial data $\left(\Phi^{0}, \Phi^{1}\right) \in H_{1 / 2}^{-} \times H^{-}$for which the solution $\Phi=(\varphi, \psi)^{T}$ of (1.3) satisfies

$$
\left\|\left(\Phi^{0}, \Phi^{1}\right)\right\|_{X_{1}}^{-2} \int_{0}^{T} \int_{\Gamma}\left(\frac{\partial \varphi}{\partial \nu}+\alpha \psi \nu_{1}\right)^{2} d \sigma d t<\epsilon .
$$

This result means that the observability inequality (1.2) does not hold on $H_{1 / 2}^{-} \times$ $H^{-}$(and thus on the whole space $H_{1 / 2} \times H$ ). This is in agreement with the result in [10], where it is shown that the lack of observability is related to the essential spectrum.

Before proving these results, we establish that the exact controllability of (1.1) is equivalent to the observability inequality (1.2). Actually, the control operator associated with system (1.1) is not bounded from the space of controls $L^{2}(\Gamma \times(0, T))$ to the state space $X_{-1}$ (the dual of $X_{1}=H_{1 / 2} \times H$ with respect to the pivot space $\left.X=H \times H_{-1 / 2}\right)$. Section 3 is devoted to the study of this control operator and the formulation of the observability inequality.

\section{Well-posedness of the controlled system.}

3.1. A Dirichlet map. In this section, we introduce and analyze the Dirichlet map $\mathcal{D}: D(\mathcal{D}) \subset L^{2}(\Gamma) \rightarrow H$ corresponding to system (1.1) defined as follows: $D(\mathcal{D})=\left\{v \in L^{2}(\Gamma), \mathcal{D} v \in H\right\}$, and for $v \in L^{2}(\Gamma)$, we denote by $\mathcal{D} v$ the solution $\theta=\left(\theta_{1}, \theta_{2}\right)^{T}$ of the abstract elliptic problem

$$
\left\{\begin{array}{ll}
\Lambda \theta=0 & \text { in } \Omega, \\
\theta_{1}=v 1_{\Gamma} & \text { on } \partial \Omega,
\end{array} \quad \Lambda=\left(\begin{array}{cc}
-\Delta & -\alpha \partial_{x} \\
\alpha \partial_{x} & a
\end{array}\right) .\right.
$$

For simplicity of notation, we set

$$
\alpha_{p, q}^{ \pm}=\frac{\pi\left(\lambda_{p, q}^{ \pm}-a+\alpha^{2}\right)}{\sqrt{\left(\lambda_{p, q}^{ \pm}-a\right)^{2}+\alpha^{2} p^{2} \pi^{2}}}, \quad \beta_{p, q}^{ \pm}=\frac{\pi\left(\lambda_{p, q}^{ \pm}-a\right)}{\sqrt{\left(\lambda_{p, q}^{ \pm}-a\right)^{2}+\alpha^{2} p^{2} \pi^{2}}} .
$$

The map $\mathcal{D}$ satisfies the following continuity property.

Proposition 3.1. For every $\epsilon \in(1 / 2,1], \mathcal{D} \in \mathcal{L}\left(H^{\epsilon}(\Gamma), H\right)$. Moreover for every $v \in D(\mathcal{D})$, we have

$$
\left\langle\mathcal{D} v, e_{p, q}^{ \pm}\right\rangle_{H}=p \frac{\alpha_{p, q}^{ \pm}}{\lambda_{p, q}^{ \pm}} v_{2, q}+q \frac{\beta_{p, q}^{ \pm}}{\lambda_{p, q}^{ \pm}} v_{1, p}, \quad\left\langle\mathcal{D} v, e_{q}\right\rangle_{H}=\frac{\alpha}{a \sqrt{2}} v_{2, q}
$$

with $v_{1, p}=2 \int_{0}^{1} v(x, 0) \sin (p \pi x) d x$ and $v_{2, q}=2 \int_{0}^{1} v(0, y) \sin (q \pi y) d y$.

Proof. Let $\epsilon \in(1 / 2,1]$ and $v \in H^{\epsilon}(\Gamma)$. Suppose that (3.1) has a solution $\theta \in H$. Using integrations by parts, we have

$$
0=\left\langle\Lambda \theta, e_{p, q}^{ \pm}\right\rangle_{H}=\left\langle\theta, A e_{p, q}^{ \pm}\right\rangle_{H}+\int_{\Gamma}\left(\frac{\partial \varphi_{p, q}^{ \pm}}{\partial \nu}+\alpha \psi_{p, q}^{ \pm} \nu_{1}\right) v d \sigma d t,
$$

where $e_{p, q}^{ \pm}=\left(\varphi_{p, q}^{ \pm}, \psi_{p, q}^{ \pm}\right)^{T}$. Since $A e_{p, q}^{ \pm}=\lambda_{p, q}^{ \pm} e_{p, q}^{ \pm}$, this yields

$$
\left\langle\theta, e_{p, q}^{ \pm}\right\rangle_{H}=p \frac{\alpha_{p, q}^{ \pm}}{\lambda_{p, q}^{ \pm}} v_{2, q}+q \frac{\beta_{p, q}^{ \pm}}{\lambda_{p, q}^{ \pm}} v_{1, p} .
$$


By the same arguments, we obtain

$$
\left\langle\theta, e_{q}\right\rangle_{H}=\frac{\alpha}{a \sqrt{2}} v_{2, q} .
$$

This proves that if (3.1) has a solution $\theta$, then this solution is unique and is written as

$$
\theta=\sum_{p, q \geq 1}\left\langle\theta, e_{p, q}^{+}\right\rangle_{H} e_{p, q}^{+}+\sum_{p, q \geq 1}\left\langle\theta, e_{p, q}^{-}\right\rangle_{H} e_{p, q}^{-}+\sum_{q \geq 1}\left\langle\theta, e_{q}\right\rangle_{H} e_{q},
$$

with $\left\langle\theta, e_{p, q}^{ \pm}\right\rangle_{H}$ and $\left\langle\theta, e_{q}\right\rangle_{H}$ as given by (3.4) and (3.5), respectively. Now, we have to check that such a $\theta$ is an element of $H$. Formula (3.4) gives

$$
\left|\left\langle\theta, e_{p, q}^{+}\right\rangle_{H}\right| \leq p^{\epsilon+1} \frac{\alpha_{p, q}^{+}}{\lambda_{p, q}^{+}} \frac{\left|v_{2, q}\right|}{p^{\epsilon}}+q^{\epsilon+1} \frac{\beta_{p, q}^{+}}{\lambda_{p, q}^{+}} \frac{\left|v_{1, p}\right|}{q^{\epsilon}} .
$$

From the asymptotic property $\lambda_{p, q}^{+} \sim\|(p, q)\| \rightarrow+\infty \mu_{p q}$, we have (see (3.2))

$$
\frac{\alpha_{p, q}^{+}}{\lambda_{p, q}^{+}} \sim \frac{\beta_{p, q}^{+}}{\lambda_{p, q}^{+}} \sim \frac{\pi}{\mu_{p q}} \text { as }\|(p, q)\| \rightarrow+\infty .
$$

Consequently, there exists a positive constant $c_{1}$ such that for all $(p, q) \in \mathbb{N}^{*} \times \mathbb{N}^{*}$,

$$
\frac{\alpha_{p, q}^{+}}{\lambda_{p, q}^{+}} \leq \frac{c_{1}}{\mu_{p q}} \text { and } \frac{\beta_{p, q}^{+}}{\lambda_{p, q}^{+}} \leq \frac{c_{1}}{\mu_{p q}}
$$

Using $0 \leq \epsilon \leq 1$ and the definition of $\mu_{p q}$ (see Notation 2.1), we immediately obtain

$$
p^{\epsilon+1} \frac{\alpha_{p, q}^{+}}{\lambda_{p, q}^{+}} \leq \frac{c_{1}}{\pi^{2}} \text { and } q^{\epsilon+1} \frac{\beta_{p, q}^{+}}{\lambda_{p, q}^{+}} \leq \frac{c_{1}}{\pi^{2}} .
$$

From (3.6) we deduce that

$$
\left|\left\langle\theta, e_{p, q}^{+}\right\rangle_{H}\right| \leq \frac{c_{1}}{\pi^{2}}\left(\frac{\left|v_{2, q}\right|}{p^{\epsilon}}+\frac{\left|v_{1, p}\right|}{q^{\epsilon}}\right) .
$$

This gives

$$
\sum_{p, q \geq 1}\left(\left\langle\theta, e_{p, q}^{+}\right\rangle_{H}\right)^{2} \leq 2\left(\frac{c_{1}}{\pi^{2}}\right)^{2} \sum_{r \geq 1} \frac{1}{r^{2 \epsilon}}\left(\sum_{q \geq 1}\left(v_{2, q}\right)^{2}+\sum_{p \geq 1}\left(v_{1, p}\right)^{2}\right)=C_{1}(\epsilon)\|v\|_{L^{2}(\Gamma)}^{2}
$$

where $C_{1}(\epsilon)=4 \frac{c_{1}^{2}}{\pi^{4}} \sum_{r \geq 1} \frac{1}{r^{2 \epsilon}}$.

Using the definition of $\lambda_{p, q}^{-}$and (3.2), we can prove that as $\|(p, q)\| \rightarrow+\infty$,

$$
\left|\alpha_{p, q}^{-}\right| \sim|\alpha| \frac{q^{2}}{p\left(p^{2}+q^{2}\right)}, \quad\left|\beta_{p, q}^{-}\right| \sim-|\alpha| \frac{p}{p^{2}+q^{2}} .
$$

Since $\left(\lambda_{p, q}^{-}\right)_{p, q}$ is bounded away from 0 , there exists a constant $c_{2}>0$ such that for every $(p, q) \in \mathbb{N}^{*} \times \mathbb{N}^{*}$

$$
\frac{\left|\alpha_{p, q}^{-}\right|}{\lambda_{p, q}^{-}} \leq c_{2} \frac{q^{2}}{p\left(p^{2}+q^{2}\right)}, \quad \frac{\left|\beta_{p, q}^{-}\right|}{\lambda_{p, q}^{-}} \leq c_{2} \frac{p}{p^{2}+q^{2}} .
$$

Copyright $($ ) by SIAM. Unauthorized reproduction of this article is prohibited. 
Since $0 \leq \epsilon \leq 1$, this implies that

$$
\frac{p^{\epsilon+1}\left|\alpha_{p, q}^{-}\right|}{q^{\epsilon} \lambda_{p, q}^{-}} \leq c_{2} \frac{p^{\epsilon} q^{2-\epsilon}}{p^{2}+q^{2}} \leq c_{2} \text { and } \frac{q^{\epsilon+1}\left|\beta_{p, q}^{-}\right|}{p^{\epsilon} \lambda_{p, q}^{-}} \leq c_{2} \frac{p^{1-\epsilon} q^{\epsilon+1}}{p^{2}+q^{2}} \leq c_{2} .
$$

Using (3.4), we deduce that

$$
\left|\left\langle\theta, e_{p, q}^{-}\right\rangle_{H}\right| \leq \frac{p^{\epsilon+1}\left|\alpha_{p, q}^{-}\right|}{q^{\epsilon} \lambda_{p, q}^{-}} \frac{q^{\epsilon}\left|v_{2, q}\right|}{p^{\epsilon}}+\frac{q^{\epsilon+1}\left|\beta_{p, q}^{-}\right|}{p^{\epsilon} \lambda_{p, q}^{-}} \frac{p^{\epsilon}\left|v_{1, p}\right|}{q^{\epsilon}} \leq c_{2}\left(\frac{q^{\epsilon}\left|v_{2, q}\right|}{p^{\epsilon}}+\frac{p^{\epsilon}\left|v_{1, p}\right|}{q^{\epsilon}}\right) .
$$

Since $v \in H^{\epsilon}(\Gamma)$, we can write from this inequality

$$
\sum_{p, q \geq 1}\left(\left\langle\theta, e_{p, q}^{-}\right\rangle_{H}\right)^{2} \leq 2 c_{2}^{2} \sum_{r \geq 1} \frac{1}{r^{2 \epsilon}}\left(\sum_{q \geq 1}\left(q^{\epsilon} v_{2, q}\right)^{2}+\sum_{p \geq 1}\left(p^{\epsilon} v_{1, p}\right)^{2}\right) \leq C_{2}(\epsilon)\|v\|_{H^{\epsilon}(\Gamma)}^{2}
$$

where $C_{2}(\epsilon)=4 c_{2}^{2} \sum_{r \geq 1} \frac{1}{r^{2 \epsilon}}$.

Besides, formula $(3.5)$ clearly gives $\left(\left\langle\theta, e_{q}\right\rangle_{H}\right)_{q \geq 1} \in \ell^{2}$, with

$$
\sum_{q \geq 1}\left(\left\langle\theta, e_{q}\right\rangle_{H}\right)^{2}=\frac{\alpha^{2}}{2 a^{2}} \sum_{q \geq 1}\left(v_{2, q}\right)^{2} \leq \frac{\alpha^{2}}{a^{2}}\|v\|_{L^{2}(\Gamma)}^{2} .
$$

Combining (3.7), (3.8), and (3.9), we obtain $\theta \in H$, with $\|\theta\|_{H} \leq C(\epsilon)\|v\|_{H^{\epsilon}(\Gamma)}$ and $C(\epsilon)=\max \left(C_{1}(\epsilon), C_{2}(\epsilon), \frac{|\alpha|}{a}\right)$.

Proposition 3.1 gives that $H^{\epsilon}(\Gamma) \subset D(\mathcal{D})$ for every $\epsilon \in(1 / 2,1]$. This implies that $\mathcal{D}: D(\mathcal{D}) \subset L^{2}(\Gamma) \rightarrow H$ is an unbounded operator with dense domain in $L^{2}(\Gamma)$. Consequently, the adjoint operator $\mathcal{D}^{*}$ of $\mathcal{D}$ is well-defined as an unbounded operator $\mathcal{D}^{*}: D\left(\mathcal{D}^{*}\right) \subset H \rightarrow L^{2}(\Gamma)$.

Proposition 3.2. $\mathcal{D}^{*}$ is given by

$$
\begin{aligned}
& D\left(\mathcal{D}^{*}\right)=\left\{g \in H, \int_{\Gamma}\left(\frac{\partial\left(A^{-1} g\right)_{1}}{\partial \nu}+\alpha\left(A^{-1} g\right)_{2} \nu_{1}\right)^{2} d \sigma<+\infty\right\}, \\
& \mathcal{D}^{*} g=\left(\frac{\partial\left(A^{-1} g\right)_{1}}{\partial \nu}+\alpha\left(A^{-1} g\right)_{2} \nu_{1}\right)_{\mid \Gamma}, \text { where } A^{-1} g=\left(\begin{array}{c}
\left(A^{-1} g\right)_{1} \\
\left(A^{-1} g\right)_{2}
\end{array}\right) .
\end{aligned}
$$

Proof. Let $v \in D(\mathcal{D})$ and $g \in H$. By the definition of the adjoint of an unbounded operator, $D\left(\mathcal{D}^{*}\right)$ is the set of the elements $g \in H$ such that $v \mapsto\langle\mathcal{D} v, g\rangle_{H}$ is a continuous linear form on $L^{2}(\Gamma)$. By $(3.3)$, we have

$$
\begin{aligned}
\langle\mathcal{D} v, g\rangle_{H}= & \sum_{p \geq 1} v_{1, p} \sum_{q \geq 1}\left(q \frac{\beta_{p, q}^{+}}{\lambda_{p, q}^{+}}\left\langle g, e_{p, q}^{+}\right\rangle_{H}+q \frac{\beta_{p, q}^{-}}{\lambda_{p, q}^{-}}\left\langle g, e_{p, q}^{-}\right\rangle_{H}\right) \\
(3.10) \quad & +\sum_{q \geq 1} v_{2, q}\left(\sum_{p \geq 1}\left(p \frac{\alpha_{p, q}^{+}}{\lambda_{p, q}^{+}}\left\langle g, e_{p, q}^{+}\right\rangle_{H}+p \frac{\alpha_{p, q}^{-}}{\lambda_{p, q}^{-}}\left\langle g, e_{p, q}^{-}\right\rangle_{H}\right)+\frac{\alpha}{a \sqrt{2}}\left\langle g, e_{q}\right\rangle_{H}\right) .
\end{aligned}
$$

This implies that $D\left(\mathcal{D}^{*}\right)$ is the set of the elements $g \in H$ such that the two following sequences are in $\ell^{2}$ :

$$
\begin{aligned}
& \left(x_{p}=\sum_{q \geq 1}\left(q \frac{\beta_{p, q}^{+}}{\lambda_{p, q}^{+}}\left\langle g, e_{p, q}^{+}\right\rangle_{H}+q \frac{\beta_{p, q}^{-}}{\lambda_{p, q}^{-}}\left\langle g, e_{p, q}^{-}\right\rangle_{H}\right)\right)_{p \geq 1}, \\
& \left(y_{q}=\sum_{p \geq 1}\left(p \frac{\alpha_{p, q}^{+}}{\lambda_{p, q}^{+}}\left\langle g, e_{p, q}^{+}\right\rangle_{H}+p \frac{\alpha_{p, q}^{-}}{\lambda_{p, q}^{-}}\left\langle g, e_{p, q}^{-}\right\rangle_{H}\right)+\frac{\alpha}{a \sqrt{2}}\left\langle g, e_{q}\right\rangle_{H}\right)_{q \geq 1} .
\end{aligned}
$$

Copyright $\odot$ by SIAM. Unauthorized reproduction of this article is prohibited. 
It is also easily seen that

$$
\left\|\frac{\partial\left(A^{-1} g\right)_{1}}{\partial \nu}+\alpha\left(A^{-1} g\right)_{2} \nu_{1}\right\|_{L^{2}(\Gamma)}^{2}=\left\|\left(x_{p}\right)_{p}\right\|_{\ell^{2}}^{2}+\left\|\left(y_{q}\right)_{q}\right\|_{\ell^{2}}^{2} .
$$

This allows us to conclude that

$$
D\left(\mathcal{D}^{*}\right)=\left\{g \in H,\left\|\frac{\partial\left(A^{-1} g\right)_{1}}{\partial \nu}+\alpha\left(A^{-1} g\right)_{2} \nu_{1}\right\|_{L^{2}(\Gamma)}<\infty\right\} .
$$

Now we suppose that $g \in D\left(\mathcal{D}^{*}\right)$. Then we can easily check that (3.10) leads to

$$
\langle\mathcal{D} v, g\rangle_{H}=\int_{\Gamma} v\left(\frac{\partial\left(A^{-1} g\right)_{1}}{\partial \nu}+\alpha\left(A^{-1} g\right)_{2} \nu_{1}\right) d \sigma
$$

This gives the formula for $\mathcal{D}^{*} g$.

Proposition 3.3. $\mathcal{D}: D(\mathcal{D}) \subset L^{2}(\Gamma) \rightarrow H$ is a closed operator on $L^{2}(\Gamma)$.

Proof. Let $\left(v_{n}\right)_{n \in \mathbb{N}}$ be a sequence in $D(\mathcal{D})$ which converges to a certain $v$ in $L^{2}(\Gamma)$ and such that $\left(\mathcal{D} v_{n}\right)_{n \in \mathbb{N}}$ converges in $H$ to an element $\theta$. Let $v_{1, p}^{n}$ and $v_{2, q}^{n}$ denote

$$
v_{1, p}^{n}=2 \int_{0}^{1} v_{n}(x, 0) \sin (p \pi x) d x, \quad v_{2, q}^{n}=2 \int_{0}^{1} v_{n}(0, y) \sin (q \pi y) d y .
$$

From the convergence of $v_{n}$ to $v$ in $L^{2}(\Gamma)$, we deduce that $v_{1, p}^{n} \rightarrow_{n \rightarrow+\infty} v_{1, p}$ for any $p \in \mathbb{N}^{*}$ and $v_{2, q}^{n} \rightarrow_{n \rightarrow+\infty} v_{2, q}$ for any $q \in \mathbb{N}^{*}$. Using (3.3) we conclude that $\left\langle\mathcal{D} v_{n}, e_{p, q}^{ \pm}\right\rangle_{H} \rightarrow_{n \rightarrow+\infty} p \frac{\alpha_{p, q}^{ \pm}}{\lambda_{p, q}^{ \pm}} v_{2, q}+q \frac{\beta_{p, q}^{ \pm}}{\lambda_{p, q}^{ \pm}} v_{1, p}$ and $\left\langle\mathcal{D} v_{n}, e_{q}\right\rangle_{H} \rightarrow_{n \rightarrow+\infty} \frac{\alpha}{a \sqrt{2}} v_{2, q}$ for every $(p, q) \in \mathbb{N}^{*} \times \mathbb{N}^{*}$.

Besides, the convergence of $\mathcal{D} v_{n}$ to $\theta$ in $H$ implies the convergence of $\left\langle\mathcal{D} v_{n}, e_{p, q}^{ \pm}\right\rangle_{H}$ (resp., $\left.\left\langle\mathcal{D} v_{n}, e_{q}\right\rangle_{H}\right)$ to $\left\langle\theta, e_{p, q}^{ \pm}\right\rangle_{H}$ (resp., $\left\langle\theta, e_{q}\right\rangle_{H}$ ) for every $(p, q) \in \mathbb{N}^{*} \times \mathbb{N}^{*}$. Therefore,

$$
\forall(p, q) \in \mathbb{N}^{*} \times \mathbb{N}^{*}\left\{\begin{array}{l}
\left\langle\theta, e_{p, q}^{ \pm}\right\rangle_{H}=p \frac{\alpha_{p, q}^{ \pm}}{\lambda_{p, q}^{ \pm}} v_{2, q}+q \frac{\beta_{p, q}^{ \pm}}{\lambda_{p, q}^{ \pm}} v_{1, p}, \\
\left\langle\theta, e_{q}\right\rangle_{H}=\frac{\alpha}{a \sqrt{2}} v_{2, q} .
\end{array}\right.
$$

From Proposition 3.1, this implies that $\theta=\mathcal{D} v$. It follows that $v \in D(\mathcal{D})$, since $\theta \in H$.

3.2. Toward an internal control problem. We introduce the following notation:

$$
X=H \times H_{-1 / 2}, \quad X_{-1}=H_{-1 / 2} \times H_{-1}, \quad X_{1}=H_{1 / 2} \times H
$$

and

$$
L: D(L) \subset X_{-1} \rightarrow X_{-1}, \quad L=\left(\begin{array}{cc}
0 & I \\
-A & 0
\end{array}\right), \quad D(L)=X .
$$

Note that the operator occurring in the definition of $L$ is the extension of $A$ from $H$ to $H_{-1}$.

In this section we transform the boundary control problem (1.1) into the familiar form of an internal control problem:

$$
\begin{cases}U^{\prime}=L U+B v & \text { in } Q_{T}, \\ U(0)=\left(u^{0}, u^{1}\right)^{T} & \text { in } \Omega\end{cases}
$$

Copyright $@$ by SIAM. Unauthorized reproduction of this article is prohibited. 
where $B$ is an unbounded control operator from $L^{2}(\Gamma)$ to $X_{-1}$.

Assume that $v$ is an element of $H^{1}([0, T], D(\mathcal{D}))$. For the moment let $Z$ denote the vector $Z=\left(u, u^{\prime}\right)^{T}-(\mathcal{D} v, 0)^{T}$, where $u$ is the solution of (1.1). Then $Z$ is a solution of

$$
Z^{\prime}=\left(\begin{array}{cc}
0 & I \\
-\Lambda & 0
\end{array}\right) Z-\left(\begin{array}{c}
\mathcal{D} v^{\prime} \\
0
\end{array}\right)
$$

Since $v \in H^{1}([0, T], D(\mathcal{D}))$, we have $\left(\mathcal{D} v^{\prime}, 0\right)^{T} \in L^{2}([0, T], D(L))$. Therefore, $Z$ is a solution of $Z^{\prime}=L Z-\left(\mathcal{D} v^{\prime}, 0\right)^{T}$ in $X_{-1}$, and the semigroup theory gives

$$
Z(t)=S(t) Z(0)-\int_{0}^{t} S(t-s)\left(\begin{array}{c}
\mathcal{D} v^{\prime}(s) \\
0
\end{array}\right) d s
$$

where $(S(t))_{t \geq 0}$ is the $C^{0}$-semigroup associated with the maximal and dissipative operator $L$. Integrating by parts in $(3.12)$ and using $(D v(s), 0)^{T} \in D(L)$, we obtain

$$
Z(t)=S(t) Z(0)-\left(\begin{array}{c}
\mathcal{D} v(t) \\
0
\end{array}\right)+S(t)\left(\begin{array}{c}
\mathcal{D} v(0) \\
0
\end{array}\right)-\int_{0}^{t} S(t-s) L\left(\begin{array}{c}
\mathcal{D} v(s) \\
0
\end{array}\right) d s .
$$

Replacing $Z(t)$ by its definition, we obtain the following expression for $\left(u(t), u^{\prime}(t)\right)^{T}$ :

$$
\left(\begin{array}{c}
u(t) \\
u^{\prime}(t)
\end{array}\right)=S(t)\left(\begin{array}{c}
u^{0} \\
u^{1}
\end{array}\right)+\int_{0}^{t} S(t-s) B v(s) d s
$$

where

$$
B=\left(\begin{array}{c}
0 \\
A \mathcal{D}
\end{array}\right): D(B) \subset L^{2}(\Gamma) \rightarrow X_{-1}
$$

is an unbounded operator with dense domain $D(B)=D(\mathcal{D})$. Formula (3.13) means that $\left(u, u^{\prime}\right)^{T}$ is the mild solution $U$ of the internal control system (3.11).

Theorem 3.4. For any $\left(u^{0}, u^{1}\right) \in X$ and any $v \in H^{1}([0, T], D(\mathcal{D}))$, system $(1.1)$ has a unique solution $u$ in $H_{-1 / 2}$. Moreover $u \in C([0, T], H) \cap C^{1}\left([0, T], H_{-1 / 2}\right) \cap$ $C^{2}\left([0, T], H_{-1}\right)$.

Proof. The proof is a straightforward consequence of the semigroup theory (see, for instance, $\left[21\right.$, Thm. 4.1.6, p. 113]). The main argument is that $B v \in H^{1}\left([0, T], X_{-1}\right)$ (since $v \in H^{1}([0, T], D(\mathcal{D}))$ ).

\section{Controllability and observability.}

4.1. Formulation of the observability inequality. We introduce the control operator $L_{T}: D\left(L_{T}\right) \subset L^{2}(\Gamma \times(0, T)) \rightarrow X$ defined by

$$
L_{T} v=\int_{0}^{T} S(T-t) B v(t) d t
$$

with domain $D\left(L_{T}\right)=\left\{v \in L^{2}(\Gamma \times(0, T)), L_{T} v \in X\right\}$. The exact boundary controllability problem for system (1.1) is the following: given $T>0$ large enough, initial data $\left(u^{0}, u^{1}\right) \in X$, and final data $\left(u_{T}^{0}, u_{T}^{1}\right) \in X$, find a control function $v$ in $D\left(L_{T}\right)$ such that the solution $u$ of system $(1.1)$ satisfies $\left(u(., T), u^{\prime}(., T)\right)=\left(u_{T}^{0}, u_{T}^{1}\right)$ in $\Omega$. Therefore, system (1.1) is exactly controllable at time $T$ if and only if the control operator $L_{T}$ is onto. 
By Theorem 3.4, $D\left(L_{T}\right)$ contains $H^{1}([0, T], D(\mathcal{D}))$, which is dense in $L^{2}(\Gamma \times(0, T))$ since $D(\mathcal{D})$ is dense in $L^{2}(\Gamma)$. Hence $D\left(L_{T}\right)$ is dense in $L^{2}(\Gamma \times(0, T))$. This allows us to compute the adjoint $L_{T}^{*}$ of $L_{T}$. Using arguments similar to those in the proof of Proposition 3.3, we can prove that $L_{T}: D\left(L_{T}\right) \subset L^{2}(\Gamma \times(0, T)) \rightarrow X$ is a closed operator. Consequently, the surjectivity of $L_{T}$ is equivalent (see, for instance, [4, Thm. II.19, p. 29]) to the existence of a positive constant $c$ such that

$$
\left\|L_{T}^{*}\left(\begin{array}{l}
\Psi^{0} \\
\Psi^{1}
\end{array}\right)\right\|_{L^{2}\left(\Sigma_{T}\right)}^{2} \geq c\left\|\left(\begin{array}{c}
\Psi^{0} \\
\Psi^{1}
\end{array}\right)\right\|_{X}^{2} \forall\left(\begin{array}{l}
\Psi^{0} \\
\Psi^{1}
\end{array}\right) \in D\left(L_{T}^{*}\right)
$$

This last inequality is also equivalent to

$$
\left\|L_{T}^{*} L\left(\begin{array}{c}
\Phi^{0} \\
\Phi^{1}
\end{array}\right)\right\|_{L^{2}\left(\Sigma_{T}\right)}^{2} \geq c\left\|\left(\begin{array}{c}
\Phi^{0} \\
\Phi^{1}
\end{array}\right)\right\|_{X_{1}}^{2} \quad \forall\left(\begin{array}{c}
\Phi^{0} \\
\Phi^{1}
\end{array}\right) \in D\left(L_{T}^{*} L\right) .
$$

Therefore, the exact controllability problem for system (1.1) relies on the "observability inequality" (4.2).

In what follows, we compute $L_{T}^{*} L$ to translate (4.2) in terms of the adjoint system (1.3) of system (1.1). By the definition of $L_{T}$ in (4.1), we have $L_{T}^{*}=B^{*} S(T-\cdot)^{*}$ so that it remains to compute the adjoint of $B$.

Lemma 4.1. $B^{*}: D\left(B^{*}\right) \subset X_{1} \rightarrow L^{2}(\Gamma)$ is given by

$$
\begin{aligned}
& D\left(B^{*}\right)=\left\{\left(\Phi^{0}, \Phi^{1}\right)^{T} \in X_{1} / \Phi^{1} \in D\left(\mathcal{D}^{*}\right)\right\}, \\
& B^{*}\left(\begin{array}{c}
\Phi^{0} \\
\Phi^{1}
\end{array}\right)=\mathcal{D}^{*} \Phi^{1} .
\end{aligned}
$$

Moreover for every $\left(\Phi^{0}, \Phi^{1}\right)^{T} \in D\left(B^{*} L\right)$, we have

$$
B^{*} L\left(\begin{array}{c}
\Phi^{0} \\
\Phi^{1}
\end{array}\right)=-\left(\frac{\partial \Phi_{1}^{0}}{\partial \nu}+\alpha \Phi_{2}^{0} \nu_{1}\right)_{\mid \Gamma}, \text { where } \Phi^{0}=\left(\Phi_{1}^{0}, \Phi_{2}^{0}\right)^{T}
$$

Proof. Let $v \in D(B)=D(\mathcal{D})$ and $\left(\Phi^{0}, \Phi^{1}\right)^{T} \in X_{1}$. We recall that since $A$ is self-adjoint in $H, L$ is skew-adjoint in $X_{-1}$ (i.e., $L^{*}=-L$ ). This allows us to write

$$
\left\langle\left(\begin{array}{c}
\Phi^{0} \\
\Phi^{1}
\end{array}\right), B v\right\rangle_{X_{1}, X_{-1}}=\left\langle-L\left(\begin{array}{c}
\Phi^{0} \\
\Phi^{1}
\end{array}\right), L^{-1} B v\right\rangle_{X}=\left\langle\left(\begin{array}{c}
-\Phi^{1} \\
A \Phi^{0}
\end{array}\right), L^{-1} B v\right\rangle_{X} .
$$

It is easily seen that $L^{-1}=\left(\begin{array}{cc}0 & -A^{-1} \\ I & 0\end{array}\right)$ so that $L^{-1} B v=\left(\begin{array}{c}-\mathcal{D} v \\ 0\end{array}\right)$. This gives

$$
\left\langle\left(\begin{array}{c}
\Phi^{0} \\
\Phi^{1}
\end{array}\right), B v\right\rangle_{X_{1}, X_{-1}}=\left\langle\Phi^{1}, \mathcal{D} v\right\rangle_{H}
$$

which leads to (4.3).

Now, if $\left(\Phi^{0}, \Phi^{1}\right)^{T} \in D\left(B^{*} L\right)$, then $B^{*} L\left(\begin{array}{c}\Phi^{0} \\ \Phi^{1}\end{array}\right)=B^{*}\left(\begin{array}{c}{ }_{-A \Phi^{0}} \\ -A\end{array}\right)=-\mathcal{D}^{*}\left(A \Phi^{0}\right)$, and Proposition 3.2 gives (4.4).

A direct consequence of Lemma 4.1 is the following.

Proposition 4.2. $L_{T}^{*} L: D\left(L_{T}^{*} L\right) \subset X_{1} \rightarrow L^{2}(\Gamma \times(0, T))$ is given by

$$
\begin{aligned}
& D\left(L_{T}^{*} L\right)=\left\{\left(\Phi^{0}, \Phi^{1}\right)^{T} \in X_{1} / \frac{\partial \varphi}{\partial \nu}+\alpha \psi \nu_{1} \in L^{2}(\Gamma \times(0, T))\right\}, \\
& L_{T}^{*} L\left(\begin{array}{c}
\Phi^{0} \\
\Phi^{1}
\end{array}\right)=-\left(\frac{\partial \varphi}{\partial \nu}+\alpha \psi \nu_{1}\right)_{\mid \Gamma},
\end{aligned}
$$

Copyright $@$ by SIAM. Unauthorized reproduction of this article is prohibited. 
where $\Phi=(\varphi, \psi)^{T}$ is the solution of the following backward adjoint system of (1.1):

$$
\begin{cases}\Phi^{\prime \prime}+A \Phi=0 & \text { in } Q_{T} \\ \Phi(., T)=\Phi^{0}, \Phi^{\prime}(., T)=\Phi^{1} & \text { in } \Omega .\end{cases}
$$

Thanks to Proposition 4.2, the observability inequality (4.2) consists of the following inequality:

$$
\forall\left(\begin{array}{c}
\Phi^{0} \\
\Phi^{1}
\end{array}\right) \in D\left(L_{T}^{*} L\right), \quad \int_{0}^{T} \int_{\Gamma}\left(\frac{\partial \varphi}{\partial \nu}+\alpha \psi \nu_{1}\right)^{2} d \sigma d t \geq c\left\|\left(\begin{array}{c}
\Phi^{0} \\
\Phi^{1}
\end{array}\right)\right\|_{X_{1}}^{2},
$$

where $\Phi=(\varphi, \psi)^{T}$ is the solution of the backward adjoint system (4.5). Consequently, we have the following characterization of the controllability.

Corollary 4.3. System (1.1) is exactly controllable at time $T$ if and only if there exists a constant $C(T)>0$ such that for all initial data $\left(\Phi^{0}, \Phi^{1}\right)^{T} \in D\left(L_{T}^{*} L\right)$, the solution $\Phi=(\varphi, \psi)^{T}$ of the adjoint system (1.3) with initial data $\left(\Phi^{0}, \Phi^{1}\right)$ satisfies the following observability inequality:

$$
\left\|\left(\Phi^{0}, \Phi^{1}\right)\right\|_{X_{1}}^{2} \leq C(T) \int_{0}^{T} \int_{\Gamma}\left(\frac{\partial \varphi}{\partial \nu}+\alpha \psi \nu_{1}\right)^{2} d \sigma d t
$$

4.2. Observability inequality in $\boldsymbol{H}_{1 / 2}^{+} \times H^{+}$: Proof of Theorem 2.3. In view of Corollary 4.3, Theorem 2.3 is proved if we prove the following result.

THEOREM 4.4. Let $\gamma=\frac{\pi \sqrt{\pi}}{4 \sqrt{2 \pi+|\alpha|}}$ and $T_{0}=\frac{2 \pi}{\gamma} \sqrt{1+2 \frac{\left(\lambda_{1,1}^{+}-a+\alpha^{2}\right)^{2}}{\left(\lambda_{1,1}^{+}-a\right)^{2}}}$. If $a \leq 2 \pi^{2}$, then for any $T>T_{0}$ there exists a positive constant $C^{+}(T)$ such that for all initial data $\left(\Phi^{0}, \Phi^{1}\right)^{T}$ in $D\left(L_{T}^{*} L\right) \cap\left(H_{1 / 2}^{+} \times H^{+}\right)$, the solution of (1.3) satisfies the following observability inequality:

$$
\left\|\left(\Phi^{0}, \Phi^{1}\right)\right\|_{X_{1}}^{2} \leq C^{+}(T) \int_{0}^{T} \int_{\Gamma}\left(\frac{\partial \varphi}{\partial \nu}+\alpha \psi \nu_{1}\right)^{2} d \sigma d t
$$

We detail the proof of Theorem 4.4 in three steps.

4.2.1. First step: The observability inequality in terms of Fourier series. From now on, we assume that $a \leq 2 \pi^{2}$ and $T>T_{0}$. Fix $\left(\Phi^{0}, \Phi^{1}\right)^{T} \in$ $D\left(L_{T}^{*} L\right) \cap\left(H_{1 / 2}^{+} \times H^{+}\right)$. By the definitions of $H_{1 / 2}^{+}$and $H^{+}, \Phi^{0}$ and $\Phi^{1}$ may be written as

$$
\Phi^{0}=\sum_{p, q \geq 1} \Phi_{p, q}^{0} e_{p, q}^{+}, \quad \Phi^{1}=\sum_{p, q \geq 1} \Phi_{p, q}^{1} e_{p, q}^{+},
$$

with $\sum_{p, q \geq 1} \lambda_{p, q}^{+}\left(\Phi_{p, q}^{0}\right)^{2}<+\infty$ and $\sum_{p, q \geq 1}\left(\Phi_{p, q}^{1}\right)^{2}<+\infty$. The solution $\Phi=(\varphi, \psi)^{T}$ of system (1.3) with initial data $\left(\Phi^{0}, \Phi^{1}\right)$ is given by

$$
\Phi(t)=\frac{1}{2} \sum_{p, q \geq 1}\left[a_{p, q} e^{i \sqrt{\lambda_{p, q}^{+}} t}+\overline{a_{p, q}} e^{-i \sqrt{\lambda_{p, q}^{+}} t}\right] e_{p, q}^{+}
$$

with the notation

$$
\forall(p, q) \in \mathbb{N}^{*} \times \mathbb{N}^{*}, \quad a_{p, q}=\Phi_{p, q}^{0}-i \frac{\Phi_{p, q}^{1}}{\sqrt{\lambda_{p, q}^{+}}} .
$$

Copyright $@$ ㅇ by SIAM. Unauthorized reproduction of this article is prohibited. 
Using the definition of $e_{p, q}^{+}$, the definition of $\alpha_{p, q}^{+}$and $\beta_{p, q}^{+}$in (3.2), and the Parseval equality, we can easily prove that

$$
\begin{aligned}
\int_{0}^{T} \int_{\Gamma}\left(\frac{\partial \varphi}{\partial \nu}+\alpha \psi \nu_{1}\right)^{2} d \sigma d t= & \frac{1}{2} \sum_{q \geq 1} \int_{0}^{T}\left|\sum_{p \geq 1} p \alpha_{p, q}^{+}\left(a_{p, q} e^{i \sqrt{\lambda_{p, q}^{+}} t}+\overline{a_{p, q}} e^{-i \sqrt{\lambda_{p, q}^{+}} t}\right)\right|^{2} d t \\
& +\frac{1}{2} \sum_{p \geq 1} \int_{0}^{T} \mid \sum_{q \geq 1} q \beta_{p, q}^{+}\left(a_{p, q} e^{i \sqrt{\lambda_{p, q}^{+}} t}+\left.\overline{a_{p, q}} e^{-i \sqrt{\lambda_{p}^{+}, q} t}\right|^{2} d t .\right.
\end{aligned}
$$

The left-hand side of (4.7) is given by

$$
\left\|\left(\Phi^{0}, \Phi^{1}\right)\right\|_{X_{1}}^{2}=\sum_{p, q \geq 1}\left(\lambda_{p, q}^{+}\left(\Phi_{p, q}^{0}\right)^{2}+\left(\Phi_{p, q}^{1}\right)^{2}\right)=\sum_{p, q \geq 1} \lambda_{p, q}^{+}\left|a_{p, q}\right|^{2} .
$$

Consequently, the observability inequality (4.7) is equivalent to the inequality

$$
\begin{aligned}
C(T) \sum_{p, q \geq 1} \lambda_{p, q}^{+}\left|a_{p, q}\right|^{2} \leq & \sum_{q \geq 1} \int_{0}^{T}\left|\sum_{p \geq 1} p \alpha_{p, q}^{+}\left(a_{p, q} e^{i \sqrt{\lambda_{p, q}^{+}} t}+\overline{a_{p, q}} e^{-i \sqrt{\lambda_{p}^{+}, q}}\right)\right|^{2} d t \\
& +\sum_{p \geq 1} \int_{0}^{T}\left|\sum_{q \geq 1} q \beta_{p, q}^{+}\left(a_{p, q} e^{i \sqrt{\lambda_{p, q}^{+}} t}+\overline{a_{p, q}} e^{-i \sqrt{\lambda_{p, q}^{+}} t}\right)\right|^{2} d t
\end{aligned}
$$

where $C(T)$ is a positive constant which does not depend on $\left(a_{p, q}\right)_{p, q}$. We recall below the main theorem of [18] for the observability of the wave equation in two spatial dimensions.

THEOREM 4.5 (Mehrenberger [18]). Let $\left(\lambda_{p q}\right)_{p, q}$ be a sequence of real numbers. If there exist $\gamma_{1}>0$ and $\gamma_{2}>0$ such that for every $p, p^{\prime}, q$, and $q^{\prime}$ in $\mathbb{N}^{*}$,

$$
\begin{aligned}
& p \leq \max \left(q, q^{\prime}\right) \Rightarrow\left|\lambda_{p q} \pm \lambda_{p q^{\prime}}\right| \geq \gamma_{1}\left|q \pm q^{\prime}\right|, \\
& q \leq \max \left(p, p^{\prime}\right) \Rightarrow\left|\lambda_{p q} \pm \lambda_{p^{\prime} q}\right| \geq \gamma_{2}\left|p \pm p^{\prime}\right|,
\end{aligned}
$$

then for any $T>2 \pi \sqrt{\frac{1}{\gamma_{1}^{2}}+\frac{1}{\gamma_{2}^{2}}}$, there exists a positive constant $C(T)$ such that

$$
\begin{aligned}
C(T) \sum_{p, q \geq 1}\left(p^{2}+q^{2}\right)\left|z_{p, q}\right|^{2} \leq & \sum_{q \geq 1} \int_{0}^{T}\left|\sum_{p \geq 1} p\left(z_{p, q} e^{i \lambda_{p q} t}+\overline{z_{p, q}} e^{-i \lambda_{p q} t}\right)\right|^{2} d t \\
& +\sum_{p \geq 1} \int_{0}^{T}\left|\sum_{q \geq 1} q\left(z_{p, q} e^{i \lambda_{p q} t}+\overline{z_{p, q}} e^{-i \lambda_{p q} t}\right)\right|^{2} d t
\end{aligned}
$$

for every complex sequence $\left(z_{p, q}\right)_{p, q}$ such that the sums involved are finite.

The use of Ingham-type methods (see $[12,13]$ ) is based on some gap properties (here given by (4.11)). In [18], to obtain the observability inequality for the wave equation, the author applies this theorem to the sequence $\lambda_{p q}=\sqrt{\mu_{p q}}$. In this case, our observability inequality (4.10) is similar to (4.12) since $\lambda_{p, q}^{+} \sim \mu_{p q}$ as $\|(p, q)\|$ tends to $+\infty$. The only dissimilarity is that we have two different sequences $x_{p, q}=\alpha_{p, q}^{+} a_{p, q}$ and $y_{p, q}=\beta_{p, q}^{+} a_{p, q}$ instead of one sequence $z_{p, q}$. Consequently, we cannot apply directly Theorem 4.5 with $\lambda_{p q}=\sqrt{\lambda_{p, q}^{+}}$to obtain the observability inequality (4.10). However, from the asymptotic property $\lambda_{p, q}^{+} \sim \mu_{p q}$ and the definition of $\alpha_{p, q}^{+}$and $\beta_{p, q}^{+}$ 
(see (3.2)) we can prove that both $x_{p, q}$ and $y_{p, q}$ are equivalent to the same term $\pi a_{p, q}$. This allows us to adapt the proof of Theorem 4.5. The key point is to prove that we have some good gap properties. To this end, we introduce the following notation.

NOTATION 4.6.

1. We denote by $\left(\Lambda_{p, q}\right)_{(p, q) \in \mathbb{Z}^{*} \times \mathbb{Z}^{*}}$ a sequence defined by

$$
\Lambda_{p, q}= \begin{cases}\sqrt{\lambda_{p, q}^{+}} & \text {if } p \geq 1 \quad \text { and } q \geq 1 \\ -\sqrt{\lambda_{p,-q}^{+}} & \text {if } p \geq 1 \quad \text { and } q \leq-1 \\ -\sqrt{\lambda_{-p, q}^{+}} & \text {if } p \leq-1 \quad \text { and } q \geq 1\end{cases}
$$

2. For $p \in \mathbb{Z}^{*}$ and $q \in \mathbb{N}^{*}$ we write

$$
x_{p, q}= \begin{cases}\alpha_{p, q}^{+} a_{p, q} & \text { if } p \geq 1 \\ -\overline{x_{-p, q}} & \text { if } p \leq-1\end{cases}
$$

3. For $p \in \mathbb{N}^{*}$ and $q \in \mathbb{Z}^{*}$ we define

$$
y_{p, q}= \begin{cases}\beta_{p, q}^{+} a_{p, q} & \text { if } q \geq 1 \\ -y_{p,-q} & \text { if } q \leq-1 .\end{cases}
$$

With Notation 4.6 we deduce from (4.9) that

$2 \int_{0}^{T} \int_{\Gamma}\left(\frac{\partial \varphi}{\partial \nu}+\alpha \psi \nu_{1}\right)^{2} d \sigma d t=\sum_{q \geq 1} \int_{0}^{T}\left|\sum_{p \in \mathbb{Z}^{*}} p x_{p, q} e^{i \Lambda_{p, q} t}\right|^{2} d t+\sum_{p \geq 1} \int_{0}^{T}\left|\sum_{q \in \mathbb{Z}^{*}} q y_{p, q} e^{i \Lambda_{p, q} t}\right|^{2} d t$

Thus the observability inequality (4.7) that we have to prove is the inequality

$$
C(T) \sum_{p, q \geq 1} \lambda_{p, q}^{+}\left|a_{p, q}\right|^{2} \leq \sum_{q \geq 1} \int_{0}^{T}\left|\sum_{p \in \mathbb{Z}^{*}} p x_{p, q} e^{i \Lambda_{p, q} t}\right|^{2} d t+\sum_{p \geq 1} \int_{0}^{T}\left|\sum_{q \in \mathbb{Z}^{*}} q y_{p, q} e^{i \Lambda_{p, q} t}\right|^{2} d t
$$

where $C(T)$ is a positive constant which does not depend on the sequence $\left(a_{p, q}\right)_{p, q}$.

4.2.2. Second step: Some gap properties. We prove the following gap properties for the sequence $\left(\Lambda_{p, q}\right)_{(p, q) \in \mathbb{Z}^{*} \times \mathbb{Z}^{*}}$.

Proposition 4.7. Let $\gamma$ be as in Theorem 4.4.

1. For all $p \in \mathbb{N}^{*}$ and all $\left(q, q^{\prime}\right) \in \mathbb{Z}^{*} \times \mathbb{Z}^{*}$ such that $p \leq \max \left(q, q^{\prime}\right)$,

$$
\left|\Lambda_{p, q}-\Lambda_{p, q^{\prime}}\right| \geq \gamma\left|q-q^{\prime}\right| .
$$

2. For all $q \in \mathbb{N}^{*}$ and all $\left(p, p^{\prime}\right) \in \mathbb{Z}^{*} \times \mathbb{Z}^{*}$ such that $q \leq \max \left(p, p^{\prime}\right)$,

$$
\left|\Lambda_{p, q}-\Lambda_{p^{\prime}, q}\right| \geq \gamma\left|p-p^{\prime}\right| .
$$

Proof. We give only the proof of the first assertion. The second can be proved in the same way by interchanging $\left(p, p^{\prime}\right)$ and $\left(q, q^{\prime}\right)$. According to the definition of $\Lambda_{p, q}$, it is sufficient to show that

- for all $p \in \mathbb{N}^{*}$ and all $\left(q, q^{\prime}\right) \in \mathbb{N}^{*} \times \mathbb{N}^{*}$ such that $p \leq \max \left(q, q^{\prime}\right)$, we have $\left|\sqrt{\lambda_{p, q}^{+}}-\sqrt{\lambda_{p, q^{\prime}}^{+}}\right| \geq \gamma\left|q-q^{\prime}\right|$,

- for all $p, q$, and $q^{\prime}$ in $\mathbb{N}^{*}$, we have $\sqrt{\lambda_{p, q}^{+}}+\sqrt{\lambda_{p, q^{\prime}}^{+}} \geq \gamma\left(q+q^{\prime}\right)$.

Copyright (C) by SIAM. Unauthorized reproduction of this article is prohibited. 
Let us first consider $p \in \mathbb{N}^{*}$ and $\left(q, q^{\prime}\right) \in \mathbb{N}^{*} \times \mathbb{N}^{*}$ such that $p \leq \max \left(q, q^{\prime}\right)$. Using the definition of $\lambda_{p, q}^{+}$(see Notation 2.1), we can easily check that

$$
\left|\lambda_{p, q}^{+}-\lambda_{p, q^{\prime}}^{+}\right|=\frac{1}{2}\left|q^{2}-q^{\prime 2}\right| \pi^{2}\left(1+\frac{\left(p^{2}+q^{2}\right) \pi^{2}-a+\left(p^{2}+q^{\prime 2}\right) \pi^{2}-a}{\sqrt{\delta_{p, q}}+\sqrt{\delta_{p, q^{\prime}}}}\right)
$$

with $\delta_{p, q}=\left(\left(p^{2}+q^{2}\right) \pi^{2}-a\right)^{2}+4 \alpha^{2} p^{2} \pi^{2}$. From the assumption $a \leq 2 \pi^{2}$, we have $\left(p^{2}+q^{2}\right) \pi^{2}-a \geq 2 \pi^{2}-a \geq 0$. Therefore, $\left|\lambda_{p, q}^{+}-\lambda_{p, q^{\prime}}^{+}\right| \geq \frac{\pi^{2}}{2}\left|q^{2}-q^{\prime 2}\right|$ and

$$
\left|\sqrt{\lambda_{p, q}^{+}}-\sqrt{\lambda_{p, q^{\prime}}^{+}}\right|=\frac{\left|\lambda_{p, q}^{+}-\lambda_{p, q^{\prime}}^{+}\right|}{\sqrt{\lambda_{p, q}^{+}}+\sqrt{\lambda_{p, q^{\prime}}^{+}}} \geq \frac{\pi^{2}}{2} \frac{q+q^{\prime}}{\sqrt{\lambda_{p, q}^{+}}+\sqrt{\lambda_{p, q^{\prime}}^{+}}}\left|q-q^{\prime}\right| .
$$

Consequently, we are reduced to bounding from below the quantity $\frac{q+q^{\prime}}{\sqrt{\lambda_{p}^{+}, q}+\sqrt{\lambda_{p, q^{+}}^{+}}}$. The inequality $\sqrt{x_{1}+x_{2}} \leq \sqrt{x_{1}}+\sqrt{x_{2}}$ easily gives $\lambda_{p, q}^{+} \leq\left(p^{2}+q^{2}\right) \pi^{2}+|\alpha| p \pi$ so that

$$
\frac{q+q^{\prime}}{\sqrt{\lambda_{p, q}^{+}}+\sqrt{\lambda_{p, q^{\prime}}^{+}}} \geq \frac{1}{\sqrt{\pi}} \frac{q+q^{\prime}}{\sqrt{\left(p^{2}+q^{2}\right) \pi+|\alpha| p}+\sqrt{\left(p^{2}+q^{\prime 2}\right) \pi+|\alpha| p}} .
$$

By assumption, $p \leq \max \left(q, q^{\prime}\right)$. Without loss of generality, we can assume that $q \leq q^{\prime}$. From (4.15) it follows that

$$
\frac{q+q^{\prime}}{\sqrt{\lambda_{p, q}^{+}}+\sqrt{\lambda_{p, q^{\prime}}^{+}}} \geq \frac{1}{\sqrt{\pi}} \frac{1+\frac{q}{q^{\prime}}}{\sqrt{\left(\frac{p^{2}}{q^{\prime 2}}+\frac{q^{2}}{q^{\prime 2}}\right) \pi+|\alpha| \frac{p}{q^{\prime 2}}}+\sqrt{\left(\frac{p^{2}}{q^{\prime 2}}+1\right) \pi+|\alpha| \frac{p}{q^{\prime 2}}}} \geq \frac{1}{\sqrt{\pi}} \frac{1}{2 \sqrt{2 \pi+|\alpha|}} .
$$

Combining this inequality with (4.14), we conclude that

$$
\left|\sqrt{\lambda_{p, q}^{+}}-\sqrt{\lambda_{p, q^{\prime}}^{+}}\right| \geq \frac{\pi \sqrt{\pi}}{4 \sqrt{2 \pi+|\alpha|}}\left|q-q^{\prime}\right|=\gamma\left|q-q^{\prime}\right| .
$$

Now, consider any $p, q$, and $q^{\prime}$ in $\mathbb{N}^{*}$. By the definition of $\lambda_{p, q}^{+}$(see Notation 2.1), it is clear that $\lambda_{p, q}^{+} \geq \frac{1}{2} \mu_{p q} \geq \frac{\pi^{2}}{2} q^{2}$, so that

$$
\sqrt{\lambda_{p, q}^{+}}+\sqrt{\lambda_{p, q^{\prime}}^{+}} \geq \frac{\pi}{\sqrt{2}}\left(q+q^{\prime}\right) \geq \gamma\left(q+q^{\prime}\right) .
$$

This ends the proof of item 1.

\subsubsection{Third step: Proof of the observability inequality.}

Notation 4.8. Given $T>T_{0}$, we denote by $k$ the function

$$
k(t):= \begin{cases}\sin \left(\frac{\pi t}{T}\right) & \text { if } 0 \leq t \leq T, \\ 0 & \text { else }\end{cases}
$$

Copyright $@$ ㅇ by SIAM. Unauthorized reproduction of this article is prohibited. 
and we define the following quantities:

$$
\begin{aligned}
& I_{1}:=\sum_{p \geq 1} \int_{0}^{T} k(t)\left|\sum_{q \geq p} q y_{p, q} e^{i \Lambda_{p, q} t}\right|^{2} d t, \quad I_{2}:=\sum_{q \geq 1} \int_{0}^{T} k(t)\left|\sum_{p \geq q} p x_{p, q} e^{i \Lambda_{p, q} t}\right|^{2} d t \\
& I_{3}:=\left|\sum_{p \geq 1} \int_{0}^{T} k(t)\left(\sum_{\substack{q \in \mathbb{Z}^{\star} \\
q<p}} q y_{p, q} e^{i \Lambda_{p, q} t}\right)\left(\sum_{\substack{q \in \mathbb{Z}^{\star} \\
q \geq p}} q \overline{y_{p, q}} e^{-i \Lambda_{p, q} t}\right) d t\right| \\
& I_{4}:=\left|\sum_{q \geq 1} \int_{0}^{T} k(t)\left(\sum_{\substack{p \in \mathbb{Z}^{\star} \\
p<q}} p x_{p, q} e^{i \Lambda_{p, q} t}\right)\left(\sum_{\substack{p \in \mathbb{Z}^{\star} \\
p \geq q}} p \overline{x_{p, q}} e^{-i \Lambda_{p, q} t}\right) d t\right|
\end{aligned}
$$

It is easily seen that the Fourier transform of $k$ is given by

$$
\widehat{k}(\xi)=e^{-i \frac{\xi T}{2}} T \sqrt{2 \pi} \frac{\cos \left(\frac{\xi T}{2}\right)}{\pi^{2}-T^{2} \xi^{2}} \quad \forall \xi \in \mathbb{R} .
$$

In particular, $|\widehat{k}|$ is an even function, and $\widehat{k}(0)=\frac{T}{\pi} \sqrt{\frac{2}{\pi}}$ is real. In the following lemma we bound from below the right-hand side of the observability inequality (4.13), using the quantities $I_{j}$ for $j=1, \ldots, 4$.

\section{LEMMA 4.9.}

$$
\sum_{q \geq 1} \int_{0}^{T}\left|\sum_{p \in \mathbb{Z}^{*}} p x_{p, q} e^{i \Lambda_{p, q} t}\right|^{2} d t+\sum_{p \geq 1} \int_{0}^{T}\left|\sum_{q \in \mathbb{Z}^{*}} q y_{p, q} e^{i \Lambda_{p, q} t}\right|^{2} d t \geq I_{1}+I_{2}-2\left(I_{3}+I_{4}\right) .
$$

Proof. If we prove that $\sum_{p \geq 1} \int_{0}^{T}\left|\sum_{q \in \mathbb{Z}^{\star}} q y_{p, q} e^{i \Lambda_{p, q} t}\right|^{2} d t \geq I_{1}-2 I_{3}$, then, replacing $p$ by $q$ and $y_{p, q}$ by $x_{p, q}$, we deduce that $\sum_{q \geq 1} \int_{0}^{T}\left|\sum_{p \in \mathbb{Z}^{\star}} p x_{p, q} e^{i \Lambda_{p, q} t}\right|^{2} d t \geq I_{2}-2 I_{4}$.

Since $0 \leq k(t) \leq 1$ for all $t \in[0, T]$, it follows that

$$
\begin{aligned}
\sum_{p \geq 1} \int_{0}^{T}\left|\sum_{q \in \mathbb{Z}^{\star}} q y_{p, q} e^{i \Lambda_{p, q} t}\right|^{2} d t & \geq \sum_{p \geq 1} \int_{0}^{T} k(t)\left|\sum_{q \in \mathbb{Z}^{\star}} q y_{p, q} e^{i \Lambda_{p, q} t}\right|^{2} d t \\
& =\sum_{p \geq 1} \int_{0}^{T} k(t)\left|\sum_{\substack{q \in \mathbb{Z}^{\star} \\
q \geq p}} q y_{p, q} e^{i \Lambda_{p, q} t}+\sum_{\substack{q \in \mathbb{Z}^{\star} \\
q<p}} q y_{p, q} e^{i \Lambda_{p, q} t}\right|^{2} d t \\
& \geq I_{1}+\sum_{p \geq 1} \int_{0}^{T} k(t)\left|\sum_{\substack{q \in \mathbb{Z}^{\star} \\
q<p}} q y_{p, q} e^{i \Lambda_{p, q} t}\right|^{2} d t-2 I_{3} \\
& \geq I_{1}-2 I_{3} . \quad \square
\end{aligned}
$$

As a consequence of Lemma 4.9, the observability inequality (4.13) will be established if we bound from below $I_{1}$ and $I_{2}$ and bound from above $I_{3}$ and $I_{4}$.

LEMMA 4.10.

$$
I_{1} \geq \frac{2 T}{\pi}\left(1-\left(\frac{2 \pi}{\gamma T}\right)^{2}\right) \sum_{p \geq 1} \sum_{q \geq p} q^{2}\left|y_{p, q}\right|^{2}
$$

Copyright (C) by SIAM. Unauthorized reproduction of this article is prohibited. 
Proof. By the definition of $I_{1}$ and simple computations, we have

$$
I_{1}=\sqrt{2 \pi} \sum_{p \geq 1} \sum_{q \geq p} \sum_{q^{\prime} \geq p} q y_{p, q} q^{\prime} \overline{y_{p, q^{\prime}}} \widehat{k}\left(\Lambda_{p, q^{\prime}}-\Lambda_{p, q}\right) .
$$

Then, using the inequality $\left|z_{1} z_{2}\right| \leq \frac{\left|z_{1}\right|^{2}+\left|z_{2}\right|^{2}}{2}$, we deduce that

$$
I_{1} \geq \sqrt{2 \pi} \sum_{p \geq 1}\left(\widehat{k}(0) \sum_{q \geq p} q^{2}\left|y_{p, q}\right|^{2}-\sum_{\substack{q \geq p \\ q}} \sum_{\substack{q^{\prime} \geq p \\ q^{\prime} \neq q}} \frac{q^{2}\left|y_{p, q}\right|^{2}+q^{\prime 2}\left|y_{p, q^{\prime}}\right|^{2}}{2}\left|\widehat{k}\left(\Lambda_{p, q^{\prime}}-\Lambda_{p, q}\right)\right|\right)
$$

Fix $p \in \mathbb{N}^{*}$. From parity of $|\widehat{k}|$ it follows that

$$
\begin{array}{r}
\sum_{\substack{q \geq p \\
q^{\prime}, \geq p \\
q^{\prime} \neq q}} \frac{q^{2}\left|y_{p, q}\right|^{2}+q^{\prime 2}\left|y_{p, q^{\prime}}\right|^{2}}{2}\left|\widehat{k}\left(\Lambda_{p, q^{\prime}}-\Lambda_{p, q}\right)\right| \\
=\sum_{q \geq p} q^{2}\left|y_{p, q}\right|^{2}\left(\sum_{\substack{q^{\prime} \geq p \\
q^{\prime \neq q}}}\left|\widehat{k}\left(\Lambda_{p, q^{\prime}}-\Lambda_{p, q}\right)\right|\right) .
\end{array}
$$

Now, consider $q \in \mathbb{Z}^{*}$ and $q^{\prime} \in \mathbb{Z}^{*}$ such that $q \geq p, q^{\prime} \geq p$, and $q^{\prime} \neq q$. Proposition 4.7 yields

$$
\left|\Lambda_{p, q^{\prime}}-\Lambda_{p, q}\right| \geq \gamma\left|q-q^{\prime}\right|>\gamma
$$

Since $T>T_{0}=\frac{2 \pi}{\gamma} \sqrt{1+2 \frac{\left(\lambda_{1,1}^{+}-a+\alpha^{2}\right)^{2}}{\left(\lambda_{1,1}^{+}-a\right)^{2}}}>\frac{\pi}{\gamma}$, we obtain $\left|\Lambda_{p, q^{\prime}}-\Lambda_{p, q}\right|>\gamma>\frac{\pi}{T}$. Using (4.16) and then (4.19), we deduce from this inequality that

$$
\begin{aligned}
\left|\widehat{k}\left(\Lambda_{p, q^{\prime}}-\Lambda_{p, q}\right)\right| & \leq \frac{T \sqrt{2 \pi}}{T^{2}\left(\Lambda_{p, q^{\prime}}-\Lambda_{p, q}\right)^{2}-\pi^{2}}=\widehat{k}(0)\left(\frac{2 \pi}{\gamma T}\right)^{2} \frac{1}{4\left(\frac{\Lambda_{p, q^{\prime}}-\Lambda_{p, q}}{\gamma}\right)^{2}-\left(\frac{2 \pi}{\gamma T}\right)^{2}} \\
& \leq \widehat{k}(0)\left(\frac{2 \pi}{\gamma T}\right)^{2} \frac{1}{4\left(q-q^{\prime}\right)^{2}-1} .
\end{aligned}
$$

Summing over $q^{\prime}$, we obtain

$$
\sum_{\substack{q^{\prime} \geq p \\ q^{\prime} \neq q}}\left|\widehat{k}\left(\Lambda_{p, q^{\prime}}-\Lambda_{p, q}\right)\right| \leq \widehat{k}(0)\left(\frac{2 \pi}{\gamma T}\right)^{2} \sum_{r \in \mathbb{Z}^{*}} \frac{1}{4 r^{2}-1}=\widehat{k}(0)\left(\frac{2 \pi}{\gamma T}\right)^{2} .
$$

Combining this inequality with (4.18) and (4.17), we conclude that

$$
I_{1} \geq \sqrt{2 \pi} \widehat{k}(0)\left(1-\left(\frac{2 \pi}{\gamma T}\right)^{2}\right) \sum_{\substack{q \geq p \\ p \geq 1}} q^{2}\left|y_{p, q}\right|^{2}=\frac{2 T}{\pi}\left(1-\left(\frac{2 \pi}{\gamma T}\right)^{2}\right) \sum_{\substack{q \geq p \\ p \geq 1}} q^{2}\left|y_{p, q}\right|^{2},
$$

which proves the lemma. 
Interchanging $p$ and $q$ and replacing $y_{p, q}$ by $x_{p, q}$, we also get

$$
I_{2} \geq \frac{2 T}{\pi}\left(1-\left(\frac{2 \pi}{\gamma T}\right)^{2}\right) \sum_{q \geq 1} \sum_{p \geq q} p^{2}\left|x_{p, q}\right|^{2}
$$

Since $\lambda_{p, q}^{+}>a$, it is easily seen that $\left|x_{p, q}\right| \geq\left|y_{p, q}\right|$ (see Notation 4.6). It follows that

$$
I_{2} \geq \frac{2 T}{\pi}\left(1-\left(\frac{2 \pi}{\gamma T}\right)^{2}\right) \sum_{q \geq 1} \sum_{p \geq q} p^{2}\left|y_{p, q}\right|^{2} .
$$

Adding this inequality to that of Lemma 4.10, we obtain

$$
\begin{aligned}
I_{1}+I_{2} & \geq \frac{2 T}{\pi}\left(1-\left(\frac{2 \pi}{\gamma T}\right)^{2}\right)\left(\sum_{p \geq 1} \sum_{q \geq p} q^{2}\left|y_{p, q}\right|^{2}+\sum_{q \geq 1} \sum_{p \geq q} p^{2}\left|y_{p, q}\right|^{2}\right) \\
& \geq \frac{T}{\pi}\left(1-\left(\frac{2 \pi}{\gamma T}\right)^{2}\right) \sum_{p, q \geq 1}\left(p^{2}+q^{2}\right)\left|y_{p, q}\right|^{2} .
\end{aligned}
$$

Replacing $y_{p, q}$ by its definition gives

$$
I_{1}+I_{2} \geq \frac{T}{\pi}\left(1-\left(\frac{2 \pi}{\gamma T}\right)^{2}\right) \sum_{p, q \geq 1}\left(p^{2}+q^{2}\right)\left|a_{p, q}\right|^{2}\left(\beta_{p, q}^{+}\right)^{2} .
$$

Now, let us bound from above $I_{3}$.

LEMMA 4.11.

$$
I_{3} \leq \frac{T}{\pi}\left(\frac{2 \pi}{\gamma T}\right)^{2} \sum_{p \geq 1} \sum_{q \geq 1} q^{2}\left|a_{p, q}\right|^{2}\left(\beta_{p, q}^{+}\right)^{2} .
$$

Proof. By the definition of $I_{3}$ (see Notation 4.8), we have

$$
\begin{aligned}
I_{3} & =\sqrt{2 \pi}\left|\sum_{p \geq 1} \sum_{\substack{q \in \mathbb{Z}^{*} q^{\prime} \geq p \\
q<p}} q y_{p, q} q^{\prime} \overline{y_{p, q^{\prime}}} \widehat{k}\left(\Lambda_{p, q^{\prime}}-\Lambda_{p, q}\right)\right| \\
& \leq \frac{\sqrt{2 \pi}}{2} \sum_{\substack { p \geq 1 \\
\begin{subarray}{c}{c \in \mathbb{Z}^{*} \\
q<p{ p \geq 1 \\
\begin{subarray} { c } { c \in \mathbb { Z } ^ { * } \\
q < p } }\end{subarray}} \sum_{q^{\prime} \geq p}\left(q^{2}\left|y_{p, q}\right|^{2}+q^{\prime 2}\left|y_{p, q^{\prime}}\right|^{2}\right)\left|\widehat{k}\left(\Lambda_{p, q^{\prime}}-\Lambda_{p, q}\right)\right| .
\end{aligned}
$$

Analysis similar to that in the proof of Lemma 4.10 shows that for all $p \in \mathbb{N}^{*}$ and all $q \in \mathbb{Z}^{*}$ such that $q<p$, we have

$$
\sum_{q^{\prime} \geq p}\left|\widehat{k}\left(\Lambda_{p, q^{\prime}}-\Lambda_{p, q}\right)\right| \leq \widehat{k}(0)\left(\frac{2 \pi}{\gamma T}\right)^{2} \sum_{q^{\prime} \geq p} \frac{1}{4\left(q-q^{\prime}\right)^{2}-1} \leq \frac{\widehat{k}(0)}{2}\left(\frac{2 \pi}{\gamma T}\right)^{2} .
$$

Therefore,

$$
\sum_{\substack{q \in \mathbb{Z}^{*} \\ q<p}} \sum_{q^{\prime} \geq p} q^{2}\left|y_{p, q}\right|^{2}\left|\widehat{k}\left(\Lambda_{p, q^{\prime}}-\Lambda_{p, q}\right)\right| \leq \frac{\widehat{k}(0)}{2}\left(\frac{2 \pi}{\gamma T}\right)^{2} \sum_{\substack{q \in \mathbb{Z}^{\star} \\ q<p}} q^{2}\left|y_{p, q}\right|^{2} .
$$

Copyright (C) by SIAM. Unauthorized reproduction of this article is prohibited. 
Similarly, we can prove that

$$
\sum_{q^{\prime} \geq p} \sum_{\substack{q \in \mathbb{Z}^{\star} \\ q<p}} q^{\prime 2}\left|y_{p, q^{\prime}}\right|^{2}\left|\widehat{k}\left(\Lambda_{p, q^{\prime}}-\Lambda_{p, q}\right)\right| \leq \frac{\widehat{k}(0)}{2}\left(\frac{2 \pi}{\gamma T}\right)^{2} \sum_{q^{\prime} \geq p} q^{\prime 2}\left|y_{p, q^{\prime}}\right|^{2} .
$$

Adding these last two inequalities, we obtain

$$
I_{3} \leq \frac{\sqrt{2 \pi}}{4} \widehat{k}(0)\left(\frac{2 \pi}{\gamma T}\right)^{2} \sum_{p \geq 1} \sum_{q \in \mathbb{Z}^{\star}} q^{2}\left|y_{p, q}\right|^{2},
$$

which gives the desired inequality after replacing $y_{p, q}$ by its definition.

Similarly,

$$
I_{4} \leq \frac{T}{\pi}\left(\frac{2 \pi}{\gamma T}\right)^{2} \sum_{p, q \geq 1} p^{2}\left|a_{p, q}\right|^{2}\left(\alpha_{p, q}^{+}\right)^{2} .
$$

Adding (4.21) and (4.22) leads to the following inequality (since $0 \leq \beta_{p, q}^{+} \leq \alpha_{p, q}^{+}$):

$$
I_{3}+I_{4} \leq \frac{T}{\pi}\left(\frac{2 \pi}{\gamma T}\right)^{2} \sum_{p, q \geq 1}\left(p^{2}+q^{2}\right)\left|a_{p, q}\right|^{2}\left(\alpha_{p, q}^{+}\right)^{2} .
$$

From (4.20) and (4.23) it follows that

$$
I_{1}+I_{2}-2\left(I_{3}+I_{4}\right) \geq \frac{T}{\pi} \sum_{p, q \geq 1}\left(p^{2}+q^{2}\right)\left|a_{p, q}\right|^{2} b_{p, q}
$$

where $\left(b_{p, q}\right)_{p, q}$ is defined by $b_{p, q}=\left(1-\left(\frac{2 \pi}{\gamma T}\right)^{2}\right)\left(\beta_{p, q}^{+}\right)^{2}-2\left(\frac{2 \pi}{\gamma T}\right)^{2}\left(\alpha_{p, q}^{+}\right)^{2}$. To bound from below $I_{1}+I_{2}-2\left(I_{3}+I_{4}\right)$ by $\sum_{p, q \geq 1} \lambda_{p, q}^{+}\left|a_{p, q}\right|^{2}$, it suffices to prove that the sequence $\left(\frac{\left(p^{2}+q^{2}\right) b_{p, q}}{\lambda_{p, q}^{+}}\right)_{p, q}$ is bounded from below. Actually,

$$
b_{p, q}=\frac{1}{T^{2}} \beta_{p, q}^{+}\left(T^{2}-\left(\frac{2 \pi}{\gamma}\right)^{2}\left(1+2 \frac{\left(\lambda_{p, q}^{+}-a+\alpha^{2}\right)^{2}}{\left(\lambda_{p, q}^{+}-a\right)^{2}}\right)\right),
$$

and it is easy to check that

$$
\sup _{(p, q) \in \mathbb{N}^{*} \times \mathbb{N}^{*}} \frac{\left(\lambda_{p, q}^{+}-a+\alpha^{2}\right)^{2}}{\left(\lambda_{p, q}^{+}-a\right)^{2}}=\frac{\left(\lambda_{1,1}^{+}-a+\alpha^{2}\right)^{2}}{\left(\lambda_{1,1}^{+}-a\right)^{2}} .
$$

Since $T>T_{0}=\frac{2 \pi}{\gamma} \sqrt{1+2 \frac{\left(\lambda_{1,1}^{+}-a+\alpha^{2}\right)^{2}}{\left(\lambda_{1,1}^{+}-a\right)^{2}}}$, this implies that $b_{p, q}>0$ for any $(p, q)$ in $\mathbb{N}^{*} \times \mathbb{N}^{*}$. Besides, from the asymptotic property $\lambda_{p, q}^{+} \sim\|(p, q)\| \rightarrow+\infty \mu_{p q}=\left(p^{2}+q^{2}\right) \pi^{2}$ we deduce that

$$
\frac{\left(p^{2}+q^{2}\right) b_{p, q}}{\lambda_{p, q}^{+}} \underset{\|(p, q)\| \rightarrow+\infty}{\sim} \frac{1}{\pi^{2}} b_{p, q}
$$

It is easily seen that $\lim _{\|(p, q)\| \rightarrow+\infty} b_{p, q}=\pi\left(1-3\left(\frac{2 \pi}{\gamma T}\right)^{2}\right)$. Since $T>T_{0}>\sqrt{3} \frac{2 \pi}{\gamma}$, it follows that $\lim _{\|(p, q)\| \rightarrow+\infty} b_{p, q}>0$. Summarizing, we have proved that $\left(\frac{\left(p^{2}+q^{2}\right) b_{p, q}}{\lambda_{p, q}^{+}}\right)_{p, q}$ 
is a positive sequence which admits a positive limit. Consequently, this sequence is uniformly bounded from below by a positive constant, denoted by $c$. It follows from (4.24) that

$$
I_{1}+I_{2}-2\left(I_{3}+I_{4}\right) \geq \pi c T \sum_{p, q \geq 1} \lambda_{p, q}^{+}\left|a_{p, q}\right|^{2},
$$

which gives inequality (4.13) (according to Lemma 4.9) and ends the proof of Theorem 4.4 .

\subsection{Lack of controllability in $\mathrm{H}^{-} \times \mathrm{H}_{-1 / 2}^{-}$.}

4.3.1. Proof of Proposition 2.4. We consider the two sequences $\left(p_{n}\right)_{n \in \mathbb{N}^{*}}$ and $\left(q_{n}\right)_{n \in \mathbb{N}^{*}}$ of positive integers given by $p_{n}=n(n+1) / 2$ and $q_{n}=n$. We denote by $\Phi_{n}=\left(\varphi_{n}, \psi_{n}\right)^{T}$ the solution of the adjoint system (1.3) with initial data $\left(\Phi_{n}^{0}, \Phi_{n}^{1}\right)=\left(e_{p_{n}, q_{n}}^{-}, 0\right) \in H_{1 / 2}^{-} \times H^{-}$. The idea is to write $\left(p_{n}, q_{n}\right)$ in the polar coordinates $\left(p_{n}, q_{n}\right)=r_{n}\left(\cos \left(\theta_{n}\right), \sin \left(\theta_{n}\right)\right)$. It is easily seen that $\lim _{n \rightarrow+\infty} r_{n}=+\infty$ and $\lim _{n \rightarrow+\infty} \theta_{n}=0$. This leads to $\lim _{n \rightarrow+\infty} \lambda_{p_{n}, q_{n}}^{-}=a-\alpha^{2}$. Since $\left\|\left(\Phi_{n}^{0}, \Phi_{n}^{1}\right)\right\|_{X_{1}}^{2}=$ $\left\|e_{p_{n}, q_{n}}^{-}\right\|_{H_{1 / 2}}^{2}=\lambda_{p_{n}, q_{n}}^{-}$and $a>\alpha^{2}$, it follows that $\lim _{n \rightarrow+\infty}\left\|\left(\Phi_{n}^{0}, \Phi_{n}^{1}\right)\right\|_{X_{1}}^{2}=a-$ $\alpha^{2}>0$. Besides, by simple computations we can prove that the solution $\Phi_{n}(t)=$ $\cos \left(\sqrt{\lambda_{p_{n}, q_{n}}^{-}} t\right) e_{p_{n}, q_{n}}^{-}$of (1.3) associated with $\left(\Phi_{n}^{0}, \Phi_{n}^{1}\right)$ satisfies $\lim _{n \rightarrow+\infty} \int_{0}^{T} \int_{\Gamma}\left(\frac{\partial \varphi_{n}}{\partial \nu}+\right.$ $\left.\alpha \psi_{n} \nu_{1}\right)^{2} d \sigma d t=0$. This contradicts the uniform observability and proves Proposition 2.4.

4.3.2. Numerical experiments. Note that the counterexample is obtained for $\left(\Phi^{0}, \Phi^{1}\right)$ composed of only one eigenfunction, for which the limit of the associated eigenvalue is $\lambda=a-\alpha^{2}$. This value is very particular because any other datum $\left(\Phi^{0}, \Phi^{1}\right)$ composed of one eigenfunction associated with $\lambda \in\left(a-\alpha^{2}, a\right]$ does not contradict the observability. The loss of observability may be exhibited by considering a (nontrivial) combination of such modes (as done in [10] using Weil sequences) in order to enhance the lack of spectral gap. Simply put, this phenomenon may be observed numerically as follows. Let $\mathbb{H}_{N}^{ \pm}$be the space of the initial data $\left(\Phi^{0}, \Phi^{1}\right)$ in $H_{1 / 2}^{ \pm} \times H^{ \pm}$spanned by $\left\{e_{p, q}^{ \pm}\right\}_{1 \leq p, q \leq N}$. If we denote by $\boldsymbol{\Phi}^{ \pm} \in \mathbb{R}^{2 N^{2}}$ the components of $\left(\Phi^{0}, \Phi^{1}\right)^{T}$ in the basis $\left\{e_{p, q}^{ \pm}\right\}_{1 \leq p, q \leq N}$, then we can write, for every $\left(\Phi^{0}, \Phi^{1}\right)$ in $\mathbb{H}_{N}^{ \pm}$,

$$
\left\|\left(\Phi^{0}, \Phi^{1}\right)\right\|_{X_{1}}^{2}=\left(\boldsymbol{A}^{ \pm} \boldsymbol{\Phi}^{ \pm}, \boldsymbol{\Phi}^{ \pm}\right)_{\mathbb{R}^{2 N^{2}}}, \quad \int_{0}^{T} \int_{\Gamma}\left(\frac{\partial \varphi}{\partial \nu}+\alpha \psi \nu_{1}\right)^{2} d \sigma d t=\left(\boldsymbol{B}^{ \pm} \boldsymbol{\Phi}^{ \pm}, \boldsymbol{\Phi}^{ \pm}\right)_{\mathbb{R}^{2 N^{2}}},
$$

where $\boldsymbol{A}^{ \pm}, \boldsymbol{B}^{ \pm} \in \mathbb{R}^{2 N^{2} \times 2 N^{2}}$ denote real symmetric matrices $\left(\boldsymbol{A}^{ \pm}\right.$is diagonal) and $(\cdot, \cdot)_{\mathbb{R}^{2 N^{2}}}$ denotes the scalar product in $\mathbb{R}^{2 N^{2}}$. On $\mathbb{H}_{N}^{ \pm}$, the observability inequality is formally written as

$$
\left(\boldsymbol{A}^{ \pm} \boldsymbol{\Phi}^{ \pm}, \boldsymbol{\Phi}^{ \pm}\right)_{\mathbb{R}^{2 N^{2}}} \leq C_{N}^{ \pm}(T)\left(\boldsymbol{B}^{ \pm} \boldsymbol{\Phi}^{ \pm}, \boldsymbol{\Phi}^{ \pm}\right)_{\mathbb{R}^{2 N^{2}}}
$$

The observability constant $C_{N}^{ \pm}(T)$, whose behavior allows us to detect the lack of observability, is the solution of the generalized eigenvalue problem

$$
C_{N}^{ \pm}(T)=\max \left\{\lambda>0, \boldsymbol{A}^{ \pm} \boldsymbol{\Phi}=\lambda \boldsymbol{B}^{ \pm} \boldsymbol{\Phi}, \boldsymbol{\Phi} \in \mathbb{R}^{2 N^{2}} \backslash\{0\}\right\} .
$$

In practice, since $\boldsymbol{A}^{ \pm}$is diagonal, it is easier to evaluate $\left(C_{N}^{ \pm}\right)^{-1}$ equal to the lowest eigenvalue of $\boldsymbol{B}^{ \pm}\left(\boldsymbol{A}^{ \pm}\right)^{-1}$. Table 4.1 gives the value of $C_{N}^{-}(T)$ for various values of $N$ and clearly exhibits the nonuniform boundedness with respect to $N$, in contrast to $C_{N}^{+}(T)$. This is in agreement with Theorem 2.3 and Proposition 2.4. 
TABLE 4.1

Evolution of the observability constant $C_{N}^{ \pm}(T)$ vs. $N$ for $(a, \alpha, T)=(4,1,3)$.

\begin{tabular}{c|c|c|c|c|c}
\hline & $N=5$ & $N=10$ & $N=20$ & $N=40$ & $N=80$ \\
\hline$C_{N}^{+}(T)$ & $5.01 \times 10^{-1}$ & $5.43 \times 10^{-1}$ & $5.71 \times 10^{-1}$ & $5.95 \times 10^{-1}$ & $6.02 \times 10^{-1}$ \\
$C_{N}^{-}(T)$ & $2.42 \times 10^{1}$ & $4.41 \times 10^{2}$ & $3.24 \times 10^{3}$ & $8.6 \times 10^{4}$ & $1.01 \times 10^{6}$ \\
\hline
\end{tabular}

\section{Concluding remarks and comments.}

5.1. A refined controllability result. We can prove that the observability inequality (4.6) remains true if we consider initial data $\left(\Phi^{0}, \Phi^{1}\right)$, which are spanned by all the $e_{p, q}^{+}$, all the $e_{q}$, and a finite number of $e_{p, q}^{-}$. More precisely, for every $N \in \mathbb{N}^{*}$ let us denote by $H^{N^{-}}$(resp., $H_{-1 / 2}^{N^{-}}, H_{1 / 2}^{N^{-}}$) the Hilbert subspace of $H$ (resp., $\left.H_{-1 / 2}, H_{1 / 2}\right)$ spanned by $\left\{e_{p, q}^{-}\right\}_{1 \leq p, q \leq N}$. By using Proposition 4.7, we can prove that if $a \leq 2 \pi^{2}$, then the new sequences $\left(\Lambda_{p, q}\right)_{(p, q) \in \mathbb{Z}^{*} \times \mathbb{N}^{*}}$ and $\left(\widetilde{\Lambda}_{p, q}\right)_{(p, q) \in \mathbb{N}^{*} \times \mathbb{Z}^{*}}$ defined by

$$
\begin{aligned}
& \Lambda_{p, q}= \begin{cases}\sqrt{a} & \text { if } p=1, \\
\sqrt{\lambda_{p-1, q}^{+}} & \text {if } 2 \leq p \leq N+1 \text { and } 1 \leq q \leq N, \\
\sqrt{\lambda_{p-N-1, q}^{-}} & \text {if } N+2 \leq p \leq 2 N+1 \text { and } 1 \leq q \leq N, \\
\sqrt{\lambda_{p-N-1, q}^{+}} & \text {if } p \geq 2 N+2 \text { and } 1 \leq q \leq N, \\
\sqrt{\lambda_{p-1, q}^{+}} & \text {if } p \geq 2 \text { and } q \geq N+1, \\
-\Lambda_{-p, q} & \text { if } p \leq-1 \text { and } q \geq 1,\end{cases} \\
& \widetilde{\Lambda}_{p, q}= \begin{cases}\sqrt{a} & \text { if } q=1, \\
\sqrt{\lambda_{p, q-1}^{+}} & \text {if } 1 \leq p \leq N \text { and } 2 \leq q \leq N+1, \\
\sqrt{\lambda_{p, q-N-1}^{-}} & \text {if } 1 \leq p \leq N \text { and } N+2 \leq q \leq 2 N+1, \\
\sqrt{\lambda_{p, q-N-1}^{+}} & \text {if } 1 \leq p \leq N \text { and } q \geq 2 N+2, \\
\sqrt{\lambda_{p-1, q}^{+}} & \text {if } p \geq N+1 \text { and } q \geq 2, \\
-\widetilde{\Lambda}_{p,-q} & \text { if } p \geq 1 \text { and } q \leq-1\end{cases}
\end{aligned}
$$

satisfy the following gap properties:

- For all $p \in \mathbb{N}^{*}$ and all $\left(q, q^{\prime}\right) \in \mathbb{Z}^{*} \times \mathbb{Z}^{*}$ such that $p \leq \max \left(q, q^{\prime}\right)$,

$$
\left|\widetilde{\Lambda}_{p, q}-\widetilde{\Lambda}_{p, q^{\prime}}\right| \geq \gamma_{N}\left|q-q^{\prime}\right|
$$

- for all $q \in \mathbb{N}^{*}$ and all $\left(p, p^{\prime}\right) \in \mathbb{Z}^{*} \times \mathbb{Z}^{*}$ such that $q \leq \max \left(p, p^{\prime}\right)$,

$$
\left|\Lambda_{p, q}-\Lambda_{p^{\prime}, q}\right| \geq \gamma_{N}\left|p-p^{\prime}\right|
$$

with a gap $\gamma_{N}$ which tends to 0 as $N$ tends to $+\infty$. Using the same methods as in section 4.2.3, we can prove that there exists a time $T_{N}>0$ (which tends to $+\infty$ with $N)$ such that for every $T>T_{N}$ and every initial data $\left(\Phi^{0}, \Phi^{1}\right)$ in $D\left(L_{T}^{*} L\right) \cap\left(\left(H_{1 / 2}^{a} \oplus\right.\right.$ $\left.\left.H_{1 / 2}^{+} \oplus H_{1 / 2}^{N^{-}}\right) \times\left(H^{a} \oplus H^{+} \oplus H^{N^{-}}\right)\right)$, the observability inequality (4.6) is satisfied. This leads to the following controllability result. 
Theorem 5.1. If $a \leq 2 \pi^{2}$, then there exists $T_{N}>0$ such that for any $T>T_{N}$, any initial data $\left(u^{0}, u^{1}\right)$, and final data $\left(u_{T}^{0}, u_{T}^{1}\right)$ belonging to $\left(H^{a} \oplus H^{+} \oplus H^{N^{-}}\right) \times$ $\left(H_{-1 / 2}^{a} \oplus H_{-1 / 2}^{+} \oplus H_{-1 / 2}^{N^{-}}\right)$, there exists a control function $v$ in $D\left(L_{T}\right)$ such that the solution $u$ of $(1.1)$ satisfies $\left(u(\cdot, T), u^{\prime}(\cdot, T)\right)=\left(u_{T}^{0}, u_{T}^{1}\right)$ in $\Omega$.

Remark 5.2. As a consequence of Theorem 5.1, $\overline{\cup_{T>0} \mathrm{R}\left(L_{T}\right)}=X$ (where $\mathrm{R}\left(L_{T}\right)$ denotes the range of $\left.L_{T}\right)$. However, the problem of approximate controllability at a fixed time $T$ (which consists of $\overline{\mathrm{R}\left(L_{T}\right)}=X$ ) is still open.

5.2. Controllability with respect to $\boldsymbol{T}$. The eigenvalue problem (4.25) allows us to estimate numerically the minimal controllability time for any $a, \alpha, N$ fixed. Figure 5.1 depicts the evolution of $C_{N}^{+}(T)$ with respect to $T$ for $(a, \alpha, N)=(4,1,50)$ and suggests that the minimal controllability time is about 2.5 . The lower bound time $T_{0}$ in Theorem 2.3 leading to $T_{0} \approx 21.96$ in the case of $(a, \alpha, N)=(4,1,50)$ is thus not sharp.

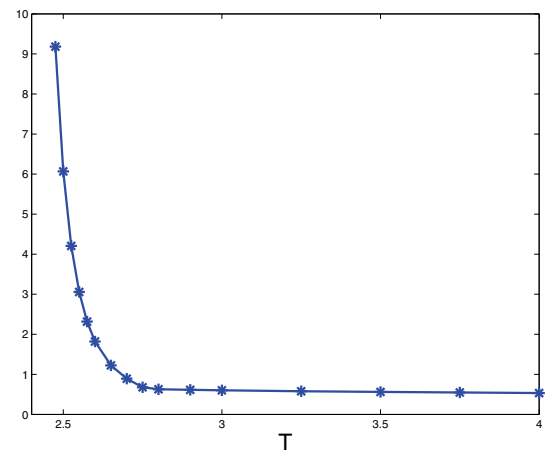

FIG. 5.1. Evolution of $C_{N}^{+}(T)$ with respect to $T$ for $(a, \alpha, N)=(4,1,50)$.

5.3. Partial controllability. Following [14], one may analyze the uniform partial controllability, which consists of driving to rest only the first component $u_{1}$ of system (1.1). This weaker controllability problem is equivalent to proving observability inequality (4.6) for initial data $\left(\Phi^{0}, \Phi^{1}\right)$ of the adjoint system (1.3) such that $\Phi_{2}^{0}=\Phi_{2}^{1}=0$. From the second equation of (1.3) with $\Phi_{2}^{0}=\Phi_{2}^{1}=0$, we express $\psi$ in terms of $\varphi$ as follows: $\psi(\cdot, t)=-\alpha \int_{0}^{t} \partial_{x} \varphi(\cdot, s) \sin (\sqrt{a}(t-s)) d s$ in $Q_{T}$. The variable $\varphi$ is then the solution of

$$
\begin{cases}\varphi^{\prime \prime}=\Delta \varphi-\alpha^{2} \int_{0}^{t} \partial_{x x} \varphi(\cdot, s) \sin (\sqrt{a}(t-s)) d s & \text { in } Q_{T} \\ \varphi=0 & \text { on } \Sigma_{T} \\ \left(\varphi(\cdot, 0), \varphi^{\prime}(\cdot, 0)\right)=\left(\Phi_{1}^{0}, \Phi_{1}^{1}\right) & \text { in } \Omega .\end{cases}
$$

The difference, with respect to the full controllability problem, is that the Fourier coefficients in $\varphi$ are all connected to each other. This should allow a compensation of the modes $\left\{e_{p, q}^{-}\right\}_{p, q}$ by the modes $\left\{e_{p, q}^{+}\right\}_{p, q}$ (we refer to [14] for the analysis on a similar system). Thus the partial controllability should be uniform with respect to the data $\left(u^{0}, u^{1}\right)$. The analysis remains to be fully written. 


\section{REFERENCES}

[1] F. V. Atkinson, H. Langer, R. Mennicken, and A. A. Shkalikov, The essential spectrum of some matrix operators, Math. Nachr., 167 (1994), pp. 5-20.

[2] F. Ammar-Khodja, G. Geymonat, And A. Münch, On the exact controllability of a system of mixed order with essential spectrum, C. R. Math. Acad. Sci. Paris, 346 (2008), pp. 629-634.

[3] F. D. Araruna and E. Zuazua, Controllability of the Kirchhoff system for beams as a limit of the Mindlin-Timoshenko system, SIAM J. Control Optim., 47 (2008), pp. 1909-1938.

[4] H. Brezis, Analyse fonctionnelle. Théorie et applications, Collect. Math. Appl. Maîtrise, Masson, Paris, 1983.

[5] E. B. Davies, Spectral Theory and Differential Operators, Cambridge Stud. Adv. Math. 42, Cambridge University Press, Cambridge, UK, 1995.

[6] G. Geymonat and G. Grubb, The essential spectrum of elliptic systems of mixed order, Math. Ann., 227 (1977), pp. 247-276.

[7] G. Geymonat And G. GrubB, Eigenvalue asymptotics for selfadjoint elliptic mixed order systems with nonempty essential spectrum, Boll. Un. Mat. Ital. B (5), 16 (1979), pp. 10321048.

[8] G. Geymonat, P. Loreti, and V. Valente, Contrôlabilité exacte d'un modèle de coque mince, C. R. Acad. Sci. Paris Sér. I Math., 313 (1991), pp. 81-86.

[9] G. Geymonat, P. Loreti, and V. Valente, Exact controllability of thin elastic hemispherical shell via harmonic analysis, in Boundary Value Problems for Partial Differential Equations and Applications, RMA Res. Notes Appl. Math. 29, Masson, Paris, 1993, pp. 379-385.

[10] G. Geymonat and V. Valente, A noncontrollability result for systems of mixed order, SIAM J. Control Optim., 39 (2000), pp. 661-672.

[11] S. W. HANSEN, Boundary control of a one-dimensional linear thermoelastic rod, SIAM J. Control Optim., 32 (1994), pp. 1052-1074.

[12] A. E. Ingham, Some trigonometrical inequalities with applications to the theory of series, Math. Z., 41 (1936), pp. 367-379.

[13] V. Komornik And P. Loreti, Fourier Series in Control Theory, Springer Monogr. Math., Springer-Verlag, New York, 2005.

[14] P. Loreti and V. Valente, Partial exact controllability for spherical membranes, SIAM J. Control Optim., 35 (1997), pp. 641-653.

[15] J. E. Lagnese And J. L. Lions, Modelling Analysis and Control of Thin Plates, Rech. Math. Appl. 6, Masson, Paris, 1988.

[16] J. L. Lions and E. Magenes, Problèmes aux limites non homogènes et applications, Vol. 1, Dunod, Paris, 1968.

[17] Z. LiU And B. RaO, A spectral approach to the indirect boundary control of a system of weakly coupled wave equations, Discrete Contin. Dyn. Syst., 23 (2009), pp. 399-414.

[18] M. Mehrenberger, An Ingham type proof for the boundary observability of a $N$-d wave equation, C. R. Math. Acad. Sci. Paris, 347 (2009), pp. 63-68.

[19] L. Rosier And P. Rouchon, On the controllability of a wave equation with structural damping, Int. J. Tomogr. Stat., 5 (2007), pp. 79-84.

20] J. Sanchez Hubert and E. Sanchez-Palencia, Vibration and Coupling of Continuous Systems. Asymptotic Theory, Springer, Berlin, 1989.

[21] M. Tucsnak and G. Weiss, Observation and Control for Operator Semigroups, BirkhäuserVerlag, Basel, 2009.

[22] E. Zuazua, Controllability of the linear system of thermoelasticity, J. Math. Pures Appl. (9), 74 (1995), pp. 291-315.

Copyright (c) by SIAM. Unauthorized reproduction of this article is prohibited. 